\title{
Enhanced stability of perovskite solar cells incorporating dopant-free crystalline Spiro- OMeTAD layers by vacuum sublimation
}

\author{
Angel Barranco*, Maria C. Lopez-Santos, Jesus Idigoras, Francisco J. Aparicio, José \\ Obrero-Pérez, Victor Lopez-Flores, Lidia Contreras-Bernal, Victor Rico, Javier Ferrer, Juan \\ P. Espinos, Ana Borras, Juan A. Anta, Juan R. Sanchez-Valencia*.
}

Dr. A. Barranco, Dr. M. C. Lopez-Santos, Dr. F. J. Aparicio, Dr. V. Lopez-Flores, Dr. V. Rico, Prof. J. P. Espinos, Dr. A. Borras, Dr. J. R. Sanchez-Valencia Instituto de Ciencia de Materiales de Sevilla (CSIC-Universidad de Sevilla), C/ Americo Vespucio 49, Seville, E-41092, Spain.

E-mail: angelbar@icmse.csic.es, jrsanchez@icmse.csic.es

Dr. J. R. Sanchez-Valencia

Departamento de Física Atómica, Molecular y Nuclear, Universidad de Sevilla, Avda. Reina Mercedes, E-41012, Seville, Spain

Dr. J. Idigoras, Dr. L. Contreras-Bernal, Prof. J.A. Anta

Área de Química Física, Universidad Pablo de Olavide, Seville, E-41013, Spain

Dr. J. Ferrer

Centro Nacional de Aceleradores (Universidad de Sevilla, CSIC, Junta de Andalucía), Av. Thomas A. Edison 7, E-41092, Seville, Spain

Keywords: perovskite solar cells, organometal halide perovskites, vacuum deposition, solid state hole conductors, Spiro-OMeTAD, stability.

The main handicap yet hindering the eventual exploitation of organometal halide perovskitebased solar cells on a real scenario basis is their poor stability under prolonged illumination, ambient conditions and increased temperatures. This article shows for the first time the vacuum processing of the most widely used solid-state hole conductor (SSHC), i.e. the SpiroOMeTAD, and how its dopant-free crystalline formation unprecedently improves the perovskite solar cell (PSC) stability under continuous illumination about two orders of magnitude with respect to the solution-processed reference and after annealing in air up to $200{ }^{\circ} \mathrm{C}$.

We demonstrate that the control over the temperature of the samples during the vacuum deposition enhances the crystallinity of the SSHC, obtaining a preferential orientation along 
the $\pi-\pi$ stacking direction. These results may settle a milestone towards the full vacuum processing of hybrid organic halide perovskite solar cells as well as light-emitting diodes (LEDs), with promising impacts on the development of durable devices. The microstructure, purity, and crystallinity of the vacuum sublimated Spiro-OMeTAD layers are fully elucidated by applying an unparalleled set of complementary characterization techniques, including SEM, XRD, GISAXS and GIWAXS, XPS and RBS.

\section{Introduction}

Organometal halide perovskites have emerged as one of the most promising photoabsorbent materials for efficient and cheap solar cells. ${ }^{[1-4]}$ The currently reported certified power conversion efficiency (PCE) for this type of cell has recently reached $25.2 \%^{[5]}$ and it has been made possible due to advances in the synthesis methods, material design, and device engineering. ${ }^{[6-10]}$ The research in this field underwent an inflexion point when the group of Miyasaka first ${ }^{[11]}$ and Park later ${ }^{[12]}$ replaced the liquid electrolyte by solid-state hole conductors (SSHC), which increased significantly the PCE and stability of the devices. From this moment on, most of the research on perovskite solar cells (PSCs) implement SSHC in the devices. The first and still most used SSHC is the organic molecule Spiro-OMeTAD [2,2',7,7'-tetrakis ( $N, N$-di-p-methoxyphenyl-amine) 9,9'-spirobifluorene]. Among the properties of this material that have led to its extended use are the high glass transition temperature (ca. $120^{\circ} \mathrm{C}$ ), the amorphous glassy nature that provides the material with optimal morphological stability and its facile processability. ${ }^{[13]}$

Whereas the use of Spiro-OMeTAD is widely spread, the undoped form of this material is reported to have very poor intrinsic hole mobility and conductivity, leading to high series resistance in the devices. ${ }^{[14-17]}$ The poor charge transport properties of Spiro-OMeTAD are solved in the literature by means of dopants. The most common additive for Spiro-OMeTAD is lithium bis(trifluoromethanesulfonyl) imide (LiTFSI), which dopes the system with $\mathrm{Li}^{+}$ 
cations, improving hole mobility up to 2 orders of magnitude. ${ }^{[13]}$ Based on previous publications about ionic dopants in disordered semiconductors, ${ }^{[18,19]}$ some authors claim a hopping mechanism in the Spiro-OMeTAD layer for the charge transport between the available sites as well as the increase of the hole density provided by the p-doping. ${ }^{[13,20]}$ Another common dopant that is usually combined with LiTFSI is 4-tert-butylpyridine (TBP). TBP is reported to improve several aspects of the Spiro-OMeTAD layers such as the homogeneity and the increase in the solubility of LiTFSI as well as preventing their accumulation and segregation. ${ }^{[13,21,22]}$ Other dopants such as the Co complex FK209 (tris(2(1H-pyrazol-1-yl)-4-tert-butylpyridine)cobalt(III) tri[bis(trifluoromethane) sulfonimide]), further increases the photovoltaic parameters when are doping the Spiro-OMeTAD layer in combination with LiTFSI and TBP, although the mechanism for this enhanced performance is still under debate. ${ }^{[13]}$

It is clear from the literature that a common strategy to enhance the charge transport properties of some SSHC is the addition of dopants in different relative concentrations. Unfortunately, one of the main challenges of perovskite-based solar cells is their stability and reproducibility, which is partially hindered by the use of dopants or additives. ${ }^{[13,14]}$ The most common issue is that ionic additives can contribute to the degradation of the perovskite film. For instance, $\mathrm{Li}^{+}$cations are highly hygroscopic and induce moisture absorption. ${ }^{[23,24]}$ The issue is so severe that several authors have aimed at the development of PSCs in a holetransport layer free configuration. ${ }^{[25,26]}$

However, and regardless of the significant number of examples that can be found in literature about Spiro-OMeTAD layers as SSHC, negligible attention has been paid to the effect of its crystalline structure. This can be understood due to the highly disordered nature of solutionprocessed films (the most widely spread) and to its amorphous-growing tendency related to the spiro-centre. ${ }^{[27,28]}$ Despite the low intrinsic hole mobility reported for dopant-free SpiroOMeTAD layers fabricated by wet approaches, the group of Bakr has recently reported 
significantly improved hole-transporting properties of pristine Spiro-OMeTAD single crystals. ${ }^{[28]}$ Their results are supported by theoretical calculations performed by Brédas and Houk, ${ }^{[29,30]}$ who reported a very high intrinsic hole conduction of crystalline Spiro-OMeTAD layers along the $\pi-\pi$ stacking direction of the crystal. Their findings demonstrated two key aspects: i) the crystalline order of the SSHC is crucial to promote new charge transport pathways and ii) the negligible effect of the lithium salts on the hole mobility of the bulk Spiro-OMeTAD. ${ }^{[28]}$ Even though these results show the potential of crystalline SpiroOMeTAD layers to enhance the photovoltaic properties of perovskite-based solar cells, their implementation is not straightforward due to the antisolvent experimental strategy used to grow this organic molecule in its crystalline form. ${ }^{[28]}$

Within such a general framework, the aim of this work is twofold, firstly, we report the unprecedented sublimation under vacuum conditions of the most widely used SSHC in perovskite solar cells, the Spiro-OMeTAD, in the form of dopant-free crystalline layers and secondly, we demonstrate the enhanced stability of the dopant-free Spiro-OMeTAD layers acting as SSHC in PSCs in comparison with the solution-processed counterpart. Our results reveal on one hand that the substrate temperature is a critical parameter controlling the microstructure and crystallinity of the layers. On the other hand, the implementation of these vacuum sublimated Spiro-OMeTAD layers on perovskite-based solar cells have demonstrated two key aspects: i) a considerably increased power conversion efficiency in comparison to the dopant-free Spiro-OMeTAD layers fabricated by a standard wet approach and ii) a significant enhancement of the stability of the cells, which have been tested under continuous illumination during 40 hours and after annealing in air up to $200{ }^{\circ} \mathrm{C}$.

\section{Results and discussion}

Spiro-OMeTAD molecules were sublimed under high vacuum on substrates at different temperatures, namely, room temperature (ca. $\left.25^{\circ} \mathrm{C}\right), 70$ and $110^{\circ} \mathrm{C}$. Figure $1 \mathrm{a}-\mathrm{c}$ ) shows the 
cross-section SEM views of Spiro-OMeTAD thin films deposited on silicon wafers. The room temperature sample (a) presents a microstructure consisting of a $50 \mathrm{~nm}$ compact layer followed by columns of ca. $50 \mathrm{~nm}$ wide and $220 \mathrm{~nm}$ long. By contrast, samples deposited at higher temperatures $\left(70\right.$ and $\left.110^{\circ} \mathrm{C}\right)$ exhibit a dense and continuous compact microstructure. It is worthy to note that for all the three different temperatures we obtained highly homogeneous thin films, with tolerance in the thickness of lower than $10 \%$ for coverage area larger than $25 \mathrm{~cm}^{2}$. With the naked eye, it can be observed that these films presented no cracks or defects and fine adhesion to reference substrates (as fused silica and $\mathrm{Si}(100)$ ) as well to the perovskite layer. These characteristics along to the dense microstructure shown in Figure $1 \mathrm{~b}-\mathrm{c}$ ) are very convenient results from the point of view of their expected performance in the final devices.

The Spiro-OMeTAD layer acts as both the hole transport layer and the interface between the perovskite and gold electrode and environment. Thus, it is expected for higher compactness of the Spiro-OMeTAD layer an enhanced performance in the protection of the perovskite to the atmospheric oxygen and moisture. On the other hand, Bakr et al. ${ }^{[28]}$ have reported the improvement in the hole mobility of crystalline Spiro-OMeTAD layers. The elucidation of the crystalline structure of organic nanocomposites and coatings is not straightforward by means of X-Ray Diffraction (XRD). ${ }^{[31]}$ This is due to several factors: i) the characteristics unit cells and planar distances are longer (in the range of nanometers) than those corresponding to the inorganic compounds and ii) the low structure factor of carbon for X-Rays. ${ }^{[31]}$ In addition, these materials usually become amorphous under the action of the electron beam, hampering their characterization by electron diffraction (SAED or EBSD). ${ }^{[32]}$ Thus, as it is expected for such an organic molecule, the grazing incidence XRD of the Spiro-OMeTAD sample deposited at $110^{\circ} \mathrm{C}$ shows no peaks in the expected range (at small angles $3-10^{\circ}$ ) as it is shown in Figure S1 in Supporting Information. 
We have tried to circumvent such a handicap by a combination of Raman spectroscopy and Grazing-Incidence Small (Wide) Angle X-Ray Scattering GISAXS (GIWAXS) analyses. For a first assessment, Figure $1 \mathrm{~d}$ ) gathers the Raman spectra for layers deposited at the three different temperatures and the corresponding to the Spiro molecules in the form of powder for the sake of comparison. It can be noted that the spectrum of Spiro-OMeTAD deposited at room temperature (RT, black line) does not show any Raman feature, indicating the amorphous nature of the film. Interestingly, the substrate temperature increase induces the crystallization of the layers, supported by the appearance of Raman bands in the same positions than the Spiro-OMeTAD molecules in their crystalline powder form (blue line). Trying to unravel the effect of the deposition temperature on the microstructure and crystallinity of the Spiro-OMeTAD layers, GISAXS and GIWAXS analyses were performed (see Experimental Methods). To our knowledge, this type of analysis has not been performed before, likely due to the amorphous nature of Spiro-OMeTAD layers prepared by wet approaches. GISAXS patterns in Figure 2 a) show a larger dispersion width for the RT SpiroOMeTAD layer indicating a more disordered structure. By contrast, the sample deposited at $110^{\circ} \mathrm{C}$ presents (besides the typical Yoneda peak maximum at ca. $0.6 \mathrm{~nm}^{-1}$ ) a qz profile along the reflection plane composed by a vertical oscillatory intensity distribution. The oscillations are caused by a resonant diffuse scattering that is linked to the thickness of a layer with correlated interfaces. ${ }^{[33]}$ In our case, the layers are deposited on flat Si wafers, indicating that the roughness is very low, in agreement with the SEM observation. In fact, the separation between the maxima of the oscillations far from the Yoneda peak is related to the layer thickness through the expression $\mathrm{d}=2 \pi / \Delta \mathrm{q}_{\mathrm{z}}{ }^{[34]}$ that results in a value of $\mathrm{d}=306 \mathrm{~nm}$, also in agreement with the thickness observed by SEM (ca. $250 \mathrm{~nm}$ ). The GIWAXS measurements in Figure 2c) show ring patterns resulting from a randomly oriented domains distribution relative to the plane of the substrate. However, when the deposition temperature increases to $110^{\circ} \mathrm{C}$, the appearance of a well-defined scattered peak along the out-of-plane direction localized at 
$\mathrm{q}_{\mathrm{z}}=4.61 \mathrm{~nm}^{-1}$ indicates that crystalline domains are highly oriented along the surface normal with an interplanar separation of $\mathrm{D}=2 \pi / \mathrm{q}_{\mathrm{z}}=1.363 \mathrm{~nm}$. According to the work of Bakr et al. ${ }^{[28]}$ who reported the diffraction of a pristine Spiro-OMeTAD single crystal, the structure is Triclininic P-1 with cell lengths of $\mathrm{a}=1.366 \mathrm{~nm}, \mathrm{~b}=1.472 \mathrm{~nm}$, and $\mathrm{c}=1.728 \mathrm{~nm}$. Although the precision of the GIWAXS technique is not high enough to distinguish between $a$ and $b$ separations (with ca. 1 Angstrom difference), we attribute these domains to an oriented $\pi-\pi$ stacking (a-axis) of the crystal structure of Spiro-OMeTAD molecules. According to literature, both from the experimental ${ }^{[28]}$ or calculation ${ }^{[29,30]}$, a closely packed $\pi-\pi$ system provides significant electronic coupling between stacked conjugated fragments. Therefore, in good agreement with these works, the observed increase in the Spiro-OMeTAD crystallinity and the particular orientation along the a-axis observed here will have a significant impact on the hole conduction properties of the Spiro-OMeTAD layers and consequently on the photovoltaic performance of the solar cells.

The crystalline growth of Spiro-OMeTAD layers induced by deposition temperature is further supported by the photoluminescence properties shown in Figure 3 a). The samples deposited at RT present the main band at $480 \mathrm{~nm}$, with a relative intense shoulder around $550 \mathrm{~nm}$. The relative intensity between these two peaks is reversed for the Spiro-OMeTAD samples prepared at $70^{\circ} \mathrm{C}$, while at $110^{\circ} \mathrm{C}$ a single band at $520 \mathrm{~nm}$ is observed. Chlorobenzene solutions of Spiro-OMeTAD molecules exhibit a single luminescence band at ca. $430 \mathrm{~nm}$ (see grey dashed line in Figure 3 a). A similar luminescence redshift has been previously observed for spiro-, poly- and oligo-fluorenes that has been ascribed to the formation of $\mathrm{H}$ - and Jaggregates through strong $\pi-\pi$ interaction between the fluorene backbone rings. ${ }^{[35,36]}$ The comparable luminescence red-shift observed for our Spiro-OMeTAD layers prepared at $70^{\circ}$ and $110^{\circ} \mathrm{C}$ further supports the GIWAXS interpretation about the crystallization along the $\pi$ $\pi$ stacking direction. 
One of the key aspects of the Spiro-OMeTAD layers prepared in this work is their purity, inherent to the vacuum sublimation methodology employed. The surface composition of the Spiro-OMeTAD samples was measured by X-Ray Photoelectron Spectroscopy (XPS) as detailed in the Experimental Section. After their fabrication, the samples were transferred to a glovebox and transported to the XPS analysis chamber under a $\mathrm{N}_{2}$ atmosphere, ensuring that the surface state of the film is not altered under room conditions. The XPS spectra show that the only elements present in the sample correspond to O, C and N (see Figure S2). Only a small contribution related to iodine can be found in the Spiro-OMeTAD sample prepared at Room Temperature, whereas the sample prepared at $110^{\circ} \mathrm{C}$ does not present any trace of this element. This small iodine content in the RT deposited films is likely arising from the residual methylammonium iodide precursor used in the same vacuum system. The quantification (see Experimental details) is shown in Table 1 and reveals the same surface composition regardless of the deposition temperature and a very similar to that expected from the chemical formula of the Spiro-OMeTAD molecule. There is a small difference in the amount of oxygen, likely due to slight oxidation of the surface. This oxidation does not increase after exposing the layers to the air (in dark) for $24 \mathrm{~h}$ (see Figure S2). In addition, the chemical state of the molecule remains unaltered for high deposition temperature (i.e. $110^{\circ} \mathrm{C}$ ) as it can be noted from the detailed XPS spectra of C, N, and O shown in Figure S2, even after exposing the sample to the atmosphere during $24 \mathrm{~h}$.

In order to reveal the elemental composition of the Spiro-OMeTAD layers along the entire thickness, Rutherford Backscattered Spectroscopy (RBS) was performed (for samples exposed to the atmosphere in dark during $24 \mathrm{~h}$ ), since XPS technique is restricted to the surface. Even if no $\mathrm{H}$ signal is produced in the RBS spectra, a 45 atomic per cent of $\mathrm{H}$ was needed to fit the simulation to the experimental results. This value is close to the theoretical value for the Spiro-OMeTAD. For the rest of the elements, again, it can be observed a very similar composition to that obtained by XPS (therefore very similar to that expected from the 
formula) for both $\mathrm{RT}$ and $110^{\circ} \mathrm{C}$ samples. The main difference is the amount of nitrogen, which is slightly higher than expected, probably caused by the low amount of this element hindering the extraction of an exact value from the spectral fitting (See spectra in Supporting Information Fig. S3). In addition, the amount of Oxygen extracted from RBS is lower than by XPS (this latter likely due to slight oxidation of the surface) but very similar to the expected theoretical value. This indicates negligible or very low oxidation of the layers along the thickness (both RT and $110^{\circ} \mathrm{C}$ ), which is expected for vacuum sublimation. This is extremely important for the solar cells since it will demonstrate that the enhanced performance of the dopant-free layers is intrinsic to the crystalline nature of the Spiro-OMeTAD layer and not due to the oxidation of the organic molecules in the presence of oxygen.

To unravel the properties of the vacuum sublimated crystalline Spiro-OMeTAD thin films, they were deposited onto solution-processed perovskite solar cells consisting of a multilayer $\mathrm{FTO} / \mathrm{TiO}_{2}$-compact/TiO2-mesoporous /Perovskite fabricated as detailed in the experimental section. It is worth to remark that from now on, the results refer to the complete solar cell. A photograph of the complete device (including vacuum sublimated Spiro-OMeTAD layer and the top gold electrode) is presented in the inset of Figure $3 \mathrm{~b}$ ). The photoluminescence spectra corresponding to the perovskite layer (band at $765 \mathrm{~nm}$ ) covered by vacuum sublimated SpiroOMeTAD films at the different temperatures studied is shown in Figure 3 b). The spectra have been obtained by illumination from the glass side (therefore minimizing the possible excitation of Spiro) and averaging from two different samples at four different positions (eight spectra in total). It can be seen how the perovskite luminescence decreases for samples containing sublimated Spiro-OMeTAD layers at 70 and $110^{\circ} \mathrm{C}$. The luminescence stems from bimolecular radiative electron-hole recombination within the perovskite film, ${ }^{[37]}$ therefore a faster hole extraction by the SSHC layer will result in a decrease of the luminescence, as it is observed for the samples deposited at 70 and $110^{\circ} \mathrm{C}^{[38]}$ 
The photocurrent density-voltage characteristics of the champion solar cells are presented in Figure 4 and the average of photovoltaic parameters (short-circuit photocurrent (JSC), open circuit photovoltage (Voc), Fill Factor (FF) and Power Conversion Efficiency (PCE)), are shown in Figure 5 and Table 2. It can be noted an overall increase for all the photovoltaic parameters with the deposition temperature of the vacuum sublimated layers, as can be easily observed in Figure 5. Full solution processed cells incorporating dopant-free Spiro-OMeTAD layers are also shown in both Figure 5 and Table 2, presenting a very poor photovoltaic behaviour, as expected. ${ }^{[15,39]}$. These dopant-free layers processed by solution are only comparable to the cells with the Spiro-OMeTAD deposited by vacuum at RT. This can be understood due to the amorphous nature of the Spiro-OMeTAD layers in these two cases and the consequent low hole mobility expected. ${ }^{[28]}$ In particular, the increase in the deposition temperature from $\mathrm{RT}$ to $70^{\circ} \mathrm{C}$ of the vacuum sublimated films produces an increase in the fill factor, from 34.4 to $53 \%$, in the Voc, from 910 to $960 \mathrm{mV}$ and in the $\mathrm{J}_{\mathrm{SC}}$, from 10.6 to 13.4 . This change in the deposition temperature is therefore reflected in a PCE increment from 3.3 to $6.8 \%$. The further increase in the deposition temperature to $110^{\circ} \mathrm{C}$ does not alter significantly the open-circuit voltage but improves the short-circuit photocurrent, the fill factor and consequently the power conversion efficiency, reaching $8.4 \%$, a significantly high value taking into account that the Spiro-OMeTAD layer is undoped. The improvement in the photovoltaic behaviour is consistent with an improved interface with the perovskite layer, ${ }^{[7,38]}$ and is likely due to increased hole mobility induced by temperature in our Spiro-OMeTAD vacuum sublimated layers. ${ }^{[40]}$ It is worth mentioning that in contrast with other works, ${ }^{[25,26]}$ our results show that the direct deposition of the metallic electrode onto the perovskite layer results in a very poor and unstable photovoltaic behaviour (see Supporting Information S4). Figure S5 in the Supporting Information shows the Photocurrent density - Voltage curves in forward and reverse scans normalized to the maximum current at $-0.1 \mathrm{~V}$ (a-d) and without normalization (e-h). The full solution-processed cells made with the doped Spiro-OMeTAD 
show the typical hysteresis curve of a MAPI-based perovskite solar cell with a higher photocurrent in the vicinity of the maximum power point. ${ }^{[41]}$ In contrast, vacuum sublimated Spiro-OMeTAD leads to a much higher hysteresis index. This can be explained by the lower capability of the dopant-free Spiro-OMeTAD to extract holes. This lower capability induces a more acute accumulation of charge at the Perovskite/Spiro-OMeTAD interface which leads to enhanced surface recombination loss and more hysteresis. ${ }^{[42]}$ In addition, Incident Photon to Current Efficiency (IPCE) curves are presented in Figure S6. IPCE spectra show lower values for the vacuum sublimated dopant-free Spiro-OMeTAD in the region when the perovskite more contributes to the total photocurrent, i.e., $400-800 \mathrm{~nm}$. This effect is a consequence of the afore-mentioned lower hole-extraction efficiency of the sublimated Spiro at the perovskite/Spiro-OMeTAD interface. On the other hand, the SpiroOMeTAD is not expected to alter the photon harvesting efficiency of the device, which yields basically the same response in the $500-800 \mathrm{~nm}$ range. However, certain variability is observed below $500 \mathrm{~nm}$, which is most probably due to partial degradation of the perovskite itself. Taking into account that the composition of the Spiro-OMeTAD layer is the same for all the samples, we can conclude that the photovoltaic performance is governed by the crystallinity of the samples. These results are in agreement with the experimental work of Bakr ${ }^{[28]}$ and from the calculations performed by Brédas or Houk, ${ }^{[29,30]}$ about the crystalline-induced intrinsic hole conduction of Spiro-OMeTAD layers.

To unravel whether the photovoltaic improvement can be induced after the vacuum sublimation of Spiro-OMeTAD layers at a certain temperature on the perovskite photoabsorbent, we have annealed to $110^{\circ} \mathrm{C}$ in high vacuum a solar cell incorporating SpiroOMeTAD sublimated at RT during 2 hours (the time needed for the deposition). Figure S7 shows the top views $(\mathrm{a}, \mathrm{c})$ and cross-sections $(\mathrm{b}, \mathrm{d})$ micrographs of the Spiro-OMeTAD layers sublimated onto a silicon wafer piece at $\mathrm{RT}(\mathrm{a}, \mathrm{b})$ and post-annealed at $110^{\circ} \mathrm{C}$ during 2 hours in vacuum $(c, d)$. It can be observed that the columnar microstructure is preserved though the 
columns appear more connected, as they were partially fused as a consequence of the annealing. In addition, the intermediate dense layer (see Figure S7 b,d) increase in thickness from around 50 to $150 \mathrm{~nm}$. Thus, from the point of view of the morphology, the post-annealed Spiro-OMeTAD layer looks like as an intermediate stage in the evolution from the columnar RT formation and the dense and homogeneous layers formed at higher substrate temperatures. Figure S8 compares the Photocurrent density - Voltage curves of solar cells incorporating the Spiro-OMeTAD vacuum sublimated layer at RT (black curve) and post-annealed in vacuum at $110^{\circ} \mathrm{C}$ (red curve). The most important change occurs in the Fill Factor, which increases significantly from 36 to $51 \%$. The photovoltage also increases $30 \mathrm{mV}$ while the photocurrent is reduced in $1 \mathrm{~mA} \mathrm{~cm}^{-2}$, producing an overall increase in the power conversion efficiency from 3.5 to $4.6 \%$. The photovoltaic enhancement can be understood if we take into account the microstructural changes observed in Figure S7. Nevertheless, we cannot relate the photovoltaic improvement only to this aspect, since it can be also due to changes in the perovskite itself or out-diffusion of ions into the Spiro-OMeTAD layer. In any case, the photovoltaic behaviour of these vacuum annealed solar cells is much lower than the cells incorporating the vacuum sublimated Spiro-OMeTAD layer at $110^{\circ} \mathrm{C}$ (with PCE of $8.4 \%$ ). As introduced above, the Achilles' Heel of the perovskite solar cells technology is the stability under ambient conditions. Looking forward to demonstrating the enhanced performance of vacuum-processed hole transport layers from such appealing point of view, we have studied the stability of perovskite solar cells incorporating solution-processed SpiroOMeTAD layers (with TBP and LiTFSI dopants) and vacuum sublimated Spiro-OMeTAD films. Figure 6 shows the evolution of JSC (a), VOC (b), Fill Factor (c) and Power Conversion Efficiency (d) under continuous illumination at laboratory ambient conditions, controlling the temperature and humidity in the ranges between $24-25^{\circ} \mathrm{C}$ and $50-60 \%$, respectively. While the PCE of solar cells incorporating solution-processed Spiro-OMeTAD layers (doped) is decreased from $13.4 \%$ to a value below $2 \%$ in three hours of illumination, the cells containing 
vacuum-deposited Spiro-OMeTAD layers present a significantly increased stability in all the photovoltaic parameters. Thus, for all the cells incorporating sublimated Spiro-OMeTAD, the PCE increases during the first 2-3 hours and then decreases slowly during the 40 hours under continuous illumination. In particular, the solar cells with Spiro-OMeTAD sublimated at $110^{\circ} \mathrm{C}$, the PCE (Figure $6 \mathrm{~d}$ ) decreases from 8.6 to $6.9 \%$, the $70^{\circ} \mathrm{C}$ from 7 to $2.7 \%$ and the RT from 3.5 to $2.8 \%$ in 40 h of illumination. Similar behaviour can be observed for the photocurrent density (a). Nevertheless, the fill factor (c) and the open-circuit voltage (b) present a different trend. While the Voc decreases continuously for the Spiro-OMeTAD sublimated at $110^{\circ} \mathrm{C}$, for the RT and $70^{\circ} \mathrm{C}$ samples the open-circuit voltage increases. In addition, the Fill Factor increases significantly for the Spiro-OMeTAD sublimated at RT, while is constant for the 70 and $110^{\circ} \mathrm{C}$ cases. These results further support the advantages of the application of vacuum-processed SSHC with controlled microstructure and crystallinity. In fact, we trust that the enhanced stability of the PSC with the Spiro-OMeTAD processed at $110^{\circ} \mathrm{C}$ (the reduction rate of PCE is two orders of magnitude lower than solution-processed) directly respond to its intrinsic features, i.e. this layer shows the highest crystallinity, compacity (low porosity) and homogeneity of the studied set. We believe that such characteristics improve the quality of both, the contact between the perovskite and SSHC layer and between the SSHC and the gold layer, at the same time that provides better insulation of the perovskite layer from the environment, delaying its degradation. The thermal stability of the perovskite solar cells is also improved by implementing vacuum sublimated Spiro-OMeTAD films. Figure S9 a-c) show the photocurrent density-voltage curves after annealing in air for $30 \mathrm{~min}$ at the indicated temperatures (from 50 to $200^{\circ} \mathrm{C}$ ). Figure S9 a) shows the thermal evolution of perovskite solar cells including solutionprocessed Spiro-OMeTAD (doped with LiTFSI and TBP) and Figure S9 b-c) the cells incorporating vacuum sublimated Spiro-OMeTAD at RT (b) and 110C (c). Figure S9 e-g) show the evolution of the photovoltaic parameters, photocurrent (e), photovoltage (f), fill 
factor (g) and PCE (h), of the J-V curves presented in a-c). While the full solution-processed solar cells depict an obvious gradual deterioration in the J-V curves with temperature, the cells with vacuum sublimated SSHC present improved stability along with a more complex behaviour. This effect can answer to two factors, namely the oxidation of the SSHC or the crystalline growth of Spiro-OMeTAD. The picture in Figure S10 allows the naked eye appreciation of the predominant role of the later factor as it clearly shows the large crystalline growth corresponding to vacuum sublimated Spiro-OMeTAD at $110^{\circ} \mathrm{C}$ after the annealing stability test carried out in air up to $200^{\circ} \mathrm{C}$.

To fully understand the physical phenomena occurring to the solar cells incorporating vacuum sublimated Spiro-OMeTAD layers, which induces important modifications in the photovoltaic behaviour, is necessary a more detailed and fundamental study. However, our results clearly show enhanced thermal stability for perovskite solar cells incorporating vacuum sublimated Spiro-OMeTAD layers. This can be clearly seen in the evolution of the photovoltaic parameters, especially in the PCE (Figure S9 h). While the PCE is drastically decreased for full solution-processed solar cells annealed at $150^{\circ} \mathrm{C}(2.4 \%)$, the ones incorporating vacuum sublimated Spiro-OMeTAD films is significantly higher (4.8 and 6.5\% for Spiro-OMeTAD synthesized at RT and $110^{\circ} \mathrm{C}$, respectively). Moreover, the annealing at $200^{\circ} \mathrm{C}$ completely degrades the full solution-processed cells, while the vacuum sublimated are still working (although with a very low PCE of 0.7 and $2.3 \%$ for Spiro-OMeTAD synthesized at RT and $110^{\circ} \mathrm{C}$, correspondently). The thermal degradation can be clearly seen in the photograph of Figure S9 d) that compares the appearance of the three cells after annealing at $200^{\circ} \mathrm{C}$ in air for 30 minutes. The full solution-processed cell appears completely degraded with the characteristic yellowish-transparent colour corresponding to the decomposition of the MAPI to $\mathrm{PbI}_{2}$. The vacuum sublimated Spiro-OMeTAD synthesized at RT is still brownish although shows a partial conversion to yellow, meanwhile, the colour of the cell with the vacuum processed at $110^{\circ} \mathrm{C} \mathrm{SSHC}$ indicates that the decomposition of the perovskite has been 
delayed. We attribute this effect to the compacity of the vacuum sublimated SSHC layer, that reduces significantly the oxygen and moisture-mediated degradation mechanisms. ${ }^{[43]}$ It is important to note that although the photovoltaic behaviour is far from the values that can be reached by using LiTFSI and TBP dopants (see Figure 5 and the last line of Table 2), this is the first example in the literature of perovskite solar cells implementing a vacuum sublimated dopant-free Spiro-OMeTAD thin film, which is the most widely used SSHC in this type of devices. We have demonstrated that the solar cells incorporating vacuum sublimated Spiro-OMeTAD layers present much higher stability than the solution-processed ones. The results provided here demonstrate for the first time that the temperature control during the vacuum sublimation of Spiro-OMeTAD molecules can be used to control the crystallization of the layers that otherwise grow as amorphous films. This crystallization induced growth increases the hole transporting properties of this SSHC, which are reflected in a highly improved stability. It is worth to note that the methodology proposed is compatible with the use of encapsulating layers either by vacuum ${ }^{[44]}$ or solution methods, ${ }^{[45]}$ that are foreseen to further enhance the stability of devices.

\section{Conclusions}

In summary, we have fabricated Spiro-OMeTAD layers by vacuum sublimation on samples at different temperatures, from RT to $110^{\circ} \mathrm{C}$. The layers present different microstructures, a porous columnar at RT and a flat, dense and compact microstructure at 70 and $110^{\circ} \mathrm{C}$. In addition, the samples deposited at higher temperatures $\left(70\right.$ and $\left.110^{\circ} \mathrm{C}\right)$ are crystalline as supported by Raman, photoluminescence, and GIWAXS. In particular, this latter characterization technique revealed an oriented crystalline growth along the substrate normal, consistent with $\pi-\pi$ stacking of the crystal. We have characterized the elemental composition of the samples by XPS and RBS, showing a high purity of the Spiro-OMeTAD films that 
showed the same elemental composition than expected from the chemical formula of the molecule.

We have implemented these vacuum sublimated Spiro-OMeTAD layers onto perovskitebased solar cells processed by solution. The solar cells presented very good photovoltaic behaviour, bearing in mind we deal with a dopant-free system. Moreover, we have demonstrated for the first time, two orders of magnitude stability improvement with respect to the solution-processed one and functional cells after annealing treatments up to $175-200{ }^{\circ} \mathrm{C}$, by using the most widely used SSHC in the literature. In fact, the comparison with the standard wet-processed dopant-free Spiro-OMeTAD layers demonstrates for the first time the potential of vacuum evaporated layers of Spiro-OMeTAD as highly stable SSHC.

Our results suggest that the incorporation of additives or dopants to the Spiro-OMeTAD layers is not strictly necessary to obtain efficient SSHC and consequently efficient perovskite solar cells. We trust our results will increase the potential of the development of full vacuumdeposited perovskite solar cells, ${ }^{[46]}$ with acknowledging advantages such as environmental friendly deposition (low processing temperatures and solventless processes), fabrication of perovskite solar cells in large areas with improved reproducibility and stability, and straightforward compatibility with industrially scalable processes.

\section{Experimental Section}

Deposition of vacuum sublimated Spiro-OMeTAD thin films: The deposition was performed in a high vacuum system, pumped down to a base pressure of $10^{-6}$ Torr. The deposition system consists of a Knudsen-type effusion cell. Spiro-OMeTAD molecules were acquired from Sigma-Aldrich and used without further purification. The molecules in powder form were placed inside the Knudsen-cell and heated to a temperature of ca. $280{ }^{\circ} \mathrm{C}$, to obtain a stable deposition rate of $1 \AA /$ s as monitored by a Quartz Crystal Monitor (QCM). The 
substrates used were fused silica slides, silicon (100) single crystal wafers and perovskite solar cells fabricated as detailed in the next section. The samples were annealed while the Spiro-OMeTAD deposition was taking place by means of a home-made heater at 70 and $110^{\circ} \mathrm{C}$.

Fabrication of perovskite solar cells: Perovskite solar cells were fabricated on FTO-coated glass (Pilkington-TEC15) patterned by laser etching. They were first cleaned using Hellmanex ${ }^{\circledR}$ solution and rinsed with deionized water and ethanol. Subsequently, they were ultrasonicated in 2-propanol and dried by using a nitrogen flow. $\mathrm{The}^{\mathrm{TiO}_{2}}$ blocking layer was deposited onto the substrates by spray pyrolysis at $450{ }^{\circ} \mathrm{C}$, using a titanium diisopropoxide bis(acetylacetonate) solution (75\% in 2-propanol, Sigma Aldrich) diluted in ethanol (1:3.5, $\mathrm{v} / \mathrm{v}$ ), with oxygen as a carrier gas. The $\mathrm{TiO}_{2}$ compact layer was then kept at $450{ }^{\circ} \mathrm{C}$ for 30 min for the formation of the anatase phase. Once the samples achieve room temperature, $\mathrm{a}^{\mathrm{TiO}_{2}}$ mesoporous layer was deposited by spin coating at $2000 \mathrm{rpm}$ during $10 \mathrm{~s}$ using a commercial $\mathrm{TiO}_{2}$ paste (Dyesol, 30NRD) diluted in ethanol (1:5, weight ratio). After drying at $100{ }^{\circ} \mathrm{C}$ for $10 \mathrm{~min}$, the $\mathrm{TiO}_{2}$ mesoporous layer was heated at $500{ }^{\circ} \mathrm{C}$ for $30 \mathrm{~min}$ and later cooled to room temperature. Subsequently, a pure methylammonium lead Iodide $\left(\mathrm{MAPbI}_{3}\right)$ solution was prepared to be deposited by spin coating using a methodology recently reported. ${ }^{[47]}$ The vacuum sublimated Spiro-OMeTAD layers were deposited onto these solution-processed layers. For comparative purposes, cells with doped and undoped Spiro-OMeTAD layer were prepared by solution process. Spiro-OMeTAD was deposited by dissolving $72.3 \mathrm{mg}$ in $1 \mathrm{~mL}$ of chlorobenzene as well as $17.5 \mu \mathrm{L}$ of a lithium bis (trifluoromethylsulphonyl)imide (LiTFSI) stock solution (520 mg of LiTFSI in $1 \mathrm{~mL}$ of acetonitrile), and $28.8 \mu \mathrm{L}$ of 4 -tertbutylpyridine (TBP). The Spiro-OMeTAD solution was spin-coated at $4000 \mathrm{rpm}$ for $30 \mathrm{~s}$. Finally, $60 \mathrm{~nm}$ of gold was deposited as a metallic contact by thermal evaporation under a vacuum level between $1 \cdot 10^{-6}$ and $1 \cdot 10^{-5}$ Torr. All the deposition processes (except vacuum processes) were carried out outside the glovebox under environmental conditions. 
Characterization of films and devices: SEM micrographs were acquired in a Hitachi S4800 working at $2 \mathrm{kV}$. The samples were deposited on $\mathrm{Si}(100)$ wafers for a correct cleavage to observe the cross-section images.

Raman spectra were measured in a LabRAM Horiba Jobin Yvon HR800 confocal microscope with a He-Ne laser $(532 \mathrm{~nm})$ working at $10 \mathrm{~mW}$ as the excitation source. The spectra were recorded through a 600 grooves/mm grating by means of a 100x Objective. The Raman spectra were calibrated with a $\operatorname{Si}(100)$ wafer.

GISAXS patterns were recorded at the P03/MiNaXS beamline of the PETRA III storage ring at DESY (Hamburg, Germany). An incident photon energy of $12.8 \mathrm{keV}$ with a beam size of $22.6 \times 23.3 \mu \mathrm{m}^{2}$ at the sample position was used for the analysis. Direct and reflected beam positions have been determined with a photodiode and a calibration standard of silver behenate. The sample to detector distance was set at $4500 \mathrm{~mm}$ and a PILATUS $300 \mathrm{~K}$ system (Dectris Ltd., Switzerland) with a pixel size of $172 \times 172 \mu \mathrm{m}^{2}$ was used as $2 \mathrm{D}$ detector. The beam impinged at an incident angle of $0.4^{\circ}$ with respect to the substrate surface.

GIWAXS measurements were performed at the Austrian SAXS Beamline 5.2L of the electron storage ring ELETTRA (Italy). For detection of the GIWAXS signal, a two-dimensional Pilatus3 1M detector system (Dectris Ltd., Switzerland) was used. X-ray energy and incident angle were $\lambda=0.154 \mathrm{~nm}$ and $0.3^{\circ}$ respectively. The samples were measured at a sample-todetector distance of $194,58 \mathrm{~mm}$. Silver behenate and para-Bromo benzoic acid standard were used to calibrate the set-up. Data were processed using the DPDAK software to extract 1D curves from the 2D scattering images. ${ }^{[48]}$ GISAXS and GIWAXS patterns, as well as their respective cuts, are presented with an intensity normalization of the colour scale representation.

Fluorescence spectra were recorded in a Jobin Yvon Fluorolog-3 spectrofluorometer using the front face configuration and grids of 4 and $2 \mathrm{~nm}$ for the excitation and emission monochromators, respectively. 
Current-voltage curves were measured under a solar simulator (ABET-Sun2000) with an AM 1.5G filter. The light intensity was calibrated at $100 \mathrm{~mW} \cdot \mathrm{cm}^{-2}$ using reference monocrystalline silicon solar cell with temperature output (ORIEL, 91150). A metal mask was used to define an active area of $0.16 \mathrm{~cm}^{2}$. The current-voltage curves were obtained in reverse scan using a scan rate of $100 \mathrm{mV} \cdot \mathrm{s}^{-1}$ and sweep delay of $20 \mathrm{~ms}$. Twelve cells ( 3 samples, 4 subcells, see inset of Figure 3b) containing the vacuum sublimated Spiro-OMeTAD layers deposited at the different temperatures were measured.

For structural characterization, X-ray diffractograms were acquired in a Panalytical X'PERT PRO instrument in a grazing-incidence configuration $\left(0.2^{\circ}\right)$ using the $\mathrm{Cu} \mathrm{K \alpha}(1.5418 \AA)$ radiation as the excitation source.

The stability curves were measured in under a solar simulator (ABET-Sun2000) with an AM 1.5G filter, using reference mono-crystalline silicon solar cell. A metal mask was used to define an active area of $0.16 \mathrm{~cm}^{2}$. Current-voltage curves were obtained every two minutes, using Keithley 2400 voltmeter controlled by software.

Incident photon-to-electron conversion efficiencies (IPCE) were measured by means of an Oriel Xenon lamp coupled to a $0.2 \mathrm{~m}$ monochromator (McPherson). The light intensity was determined as a function of the wavelength using a calibrated silicon photodiode $(\mathrm{PH}-100 \mathrm{Si}$,

\section{GENTECE).}

The annealing of the solar cells for the thermal stability study was performed in air, with consecutive treatments at increasing temperatures with a duration of $30 \mathrm{~min}$ each.

XPS measurements were performed in a Phoibos 100 DLD X-ray spectrometer from SPECS. The spectra were collected in the pass energy constant mode at a value of $50 \mathrm{eV}$. A Mg Ka unmonochromatized light was used as X-ray excitation source. N1s signal at $399.8 \mathrm{eV}$ was used to calibrate the binding energy scale.

Shirley type backgrounds were used to determine the area under the photoemission peaks. Later on, these raw areas were corrected for the transmission function of the analyser, the 
photoionization cross-sections of each electronic level and, finally, the escape depth of photoelectrons, in order to determine the surface atomic composition. ${ }^{[49]}$ Rutherford backscattering spectroscopy (RBS) was performed at the $3 \mathrm{MV}$ tandem accelerator of the National Center for Accelerators (Sevilla, Spain) with an alpha beam of $2.0 \mathrm{MeV}$ and a passivated implanted planar silicon (PIPS) detector at a $165^{\circ}$ scattering angle. The RBS spectra were analyzed using the SIMNRA software. ${ }^{[50]}$ Samples for these measurements were deposited over a glassy carbon substrate provided by Goodfellow. A thin Au interlayer (40 $\mathrm{nm})$ was grown by thermal evaporation between the substrate and the Spiro-OMeTAD layer to separate signals of $\mathrm{C}$ from the substrate and from the Spiro-OMeTAD layer in the RBS spectra.

\section{Supporting Information}

Supporting Information is available from the Wiley Online Library.

\section{Acknowledgements}

We thank the AEI, "Conserjería de Economía y Conocimiento de la Junta de Andalucía", MINECO (MAT2016-79866-R, MAT2013-42900-P, FPA2016-77689-C2-1-R and MAT2016-76892-C3-2-R) the EU through cohesion fund and FEDER 2014-2020 programs for financial support. J.R.S.-V. and A.Ba. acknowledge the EU project PlasmaPerovSol and funding from the European Union's Horizon 2020 research and innovation programme under the Marie Skłodowska-Curie grant agreement ID 661480. J.R.S-V thanks the University of Seville through the VI PPIT-US.

Beam times at DESY (Hamburg, Germany) and at the Elettra ring (Trieste, Italy) are acknowledged, respectively. We acknowledge DESY (Hamburg, Germany), a member of the Helmholtz Association HGF, for the provision of experimental facilities. Parts of this research were carried out at PETRA III, at the P03/MinaXS beamline and we would like to thank Dr. 
Björn Beyersdorff for assistance during GISAXS measurements. This research has received funding from the EU-H2020 research and innovation programme under grant agreement No 654360 having benefitted from the access provided by Technische Universität Graz at Elettra - TUG in Trieste (IT) within the framework on the NFFA Europe Transnational Access Activity. We thank Prof. Dr Heinz Amenitsch for the technical support during GIWAXS measurements. F.J.A and J.R.S-V acknowledge the "Juan de la Cierva" and "Ramon y Cajal" national programs, respectively. We also thank "Servicio de Microscopia Electrónica de la Universidad Pablo de Olavide".

\section{References}

[1] G. Xing, N. Mathews, S. Sun, S. S. Lim, Y. M. Lam, M. Grätzel, S. Mhaisalkar, T. C. Sum, Science 2013, 342, 344.

[2] J.-P. Correa-Baena, M. Saliba, T. Buonassisi, M. Grätzel, A. Abate, W. Tress, A. Hagfeldt, Science 2017, 358, 739.

[3] J. S. Manser, M. I. Saidaminov, J. A. Christians, O. M. Bakr, P. V. Kamat, Acc. Chem. Res. 2016, 49, 330.

[4] M. Saliba, J.-P. Correa-Baena, M. Grätzel, A. Hagfeldt, A. Abate, Angewandte Chemie International Edition 2018, 57, 2554.

[5] "Best Research-Cell Efficiencies (NREL, accessed 03 May 2019); https://www.nrel.gov/pv/assets/pdfs/best-research-cell-efficiencies-190416.pdf,"

[6] W. S. Yang, B.-W. Park, E. H. Jung, N. J. Jeon, Y. C. Kim, D. U. Lee, S. S. Shin, J. Seo, E. K. Kim, J. H. Noh, S. I. Seok, Science 2017, 356, 1376.

[7] H. Zhou, Q. Chen, G. Li, S. Luo, T. Song, H.-S. Duan, Z. Hong, J. You, Y. Liu, Y. Yang, Science 2014, 345, 542.

[8] J. Burschka, N. Pellet, S.-J. Moon, R. Humphry-Baker, P. Gao, M. K. Nazeeruddin, M. Grätzel, Nature 2013, 499, 316.

[9] N. J. Jeon, J. H. Noh, Y. C. Kim, W. S. Yang, S. Ryu, S. I. Seok, Nature Materials 2014, $13,897$.

[10] H. J. Snaith, J. Phys. Chem. Lett. 2013, 4, 3623.

[11] A. Kojima, K. Teshima, Y. Shirai, T. Miyasaka, Meet. Abstr. 2008, MA2008-02, 27.

[12] H.-S. Kim, C.-R. Lee, J.-H. Im, K.-B. Lee, T. Moehl, A. Marchioro, S.-J. Moon, R. Humphry-Baker, J.-H. Yum, J. E. Moser, M. Grätzel, N.-G. Park, Sci Rep 2012, 2, 591.

[13] Z. Hawash, L. K. Ono, Y. Qi, Advanced Materials Interfaces 2018, 5, 1700623.

[14] S. Ameen, M. A. Rub, S. A. Kosa, K. A. Alamry, M. S. Akhtar, H.-S. Shin, H.-K. Seo, A. M. Asiri, M. K. Nazeeruddin, ChemSusChem 2016, 9, 10.

[15] W. H. Nguyen, C. D. Bailie, E. L. Unger, M. D. McGehee, J. Am. Chem. Soc. 2014, 136, 10996.

[16] A. G. MacDiarmid, Angew. Chem. Int. Ed. Engl. 2001, 40, 2581.

[17] T. Leijtens, J. Lim, J. Teuscher, T. Park, H. J. Snaith, Advanced Materials 2013, 25, 3227. 
[18] V. I. Arkhipov, P. Heremans, E. V. Emelianova, H. Bässler, Physical Review B 2005, $71,045214$.

[19] H. Shimotani, G. Diguet, Y. Iwasa, Applied Physics Letters 2005, 86, 022104.

[20] H. J. Snaith, M. Grätzel, Applied Physics Letters 2006, 89, 262114.

[21] E. J. Juarez-Perez, M. R. Leyden, S. Wang, L. K. Ono, Z. Hawash, Y. Qi, Chemistry of Materials 2016, 28, 5702.

[22] S. Wang, M. Sina, P. Parikh, T. Uekert, B. Shahbazian, A. Devaraj, Y. S. Meng, Nano Letters 2016, 16, 5594.

[23] J. Luo, C. Jia, Z. Wan, F. Han, B. Zhao, R. Wang, Journal of Power Sources 2017, 342, 886.

[24] W. Zhou, Z. Wen, P. Gao, Advanced Energy Materials 2018, 8, 1702512.

[25] Y. Liu, S. Ji, S. Li, W. He, K. Wang, H. Hu, C. Ye, J. Mater. Chem. A 2015, 3, 14902.

[26] J. Shi, Y. Luo, H. Wei, J. Luo, J. Dong, S. Lv, J. Xiao, Y. Xu, L. Zhu, X. Xu, H. Wu, D. Li, Q. Meng, ACS Appl. Mater. Interfaces 2014, 6, 9711.

[27] U. Bach, D. Lupo, P. Comte, J. E. Moser, F. Weissörtel, J. Salbeck, H. Spreitzer, M. Grätzel, Nature 1998, 395, 583.

[28] D. Shi, X. Qin, Y. Li, Y. He, C. Zhong, J. Pan, H. Dong, W. Xu, T. Li, W. Hu, J.-L. Brédas, O. M. Bakr, Science Advances 2016, 2, e1501491.

[29] I. Yavuz, K. N. Houk, J. Phys. Chem. C, 2017, 121, 993-999.

[30] Y. Li, H. Li, C. Zhong, G. Sini, J.-L. Brédas, npj Flexible Electronics 2017, 1, 2.

[31] N. Widjonarko, Coatings 2016, 6, 54.

[32] A. Borras, O. Gröning, M. Aguirre, F. Gramm, P. Gröning, Langmuir 2010, 26, 5763.

[33] V. Soltwisch, A. Haase, J. Wernecke, J. Probst, M. Schoengen, S. Burger, M. Krumrey, F. Scholze, Physical Review B 2016, 94, 035419.

[34] A. Meyer, N. Franz, H. P. Oepen, J. Perlich, G. Carbone, T. H. Metzger, Nano Res. 2017, 10, 456.

[35] C. C. Wu, Y. T. Lin, H. H. Chiang, T. Y. Cho, C. W. Chen, K. T. Wong, Y. L. Liao, G. H. Lee, S. M. Peng, Applied Physics Letters 2002, 81, 577.

[36] Y. Deng, W. Yuan, Z. Jia, G. Liu, J. Phys. Chem. B 2014, 118, 14536.

[37] L. M. Herz, Annu. Rev. Phys. Chem. 2016, 67, 65.

[38] M. Salado, J. Idigoras, L. Calio, S. Kazim, M. K. Nazeeruddin, J. A. Anta, S. Ahmad, ACS Appl. Mater. Interfaces 2016, 8, 34414.

[39] H. Xi, S. Tang, X. Ma, J. Chang, D. Chen, Z. Lin, P. Zhong, H. Wang, C. Zhang, ACS Omega 2017, 2, 326.

[40] C. Chen, W. Zhang, J. Cong, M. Cheng, B. Zhang, H. Chen, P. Liu, R. Li, M. Safdari, L. Kloo, L. Sun, ACS Energy Letters 2017, 2, 497.

[41] W. Tress, N. Marinova, T. Moehl, S. M. Zakeeruddin, M. K. Nazeeruddin, M. Grätzel, Energy Environ. Sci. 2015, 8, 995.

[42] J. Idígoras, L. Contreras $\square$ Bernal, J. M. Cave, N. E. Courtier, Á. Barranco, A. Borras, J. R. Sánchez $\square$ Valencia, J. A. Anta, A. B. Walker, Advanced Materials Interfaces 2018, 5, 1801076.

[43] T. T. Ava, A. Al Mamun, S. Marsillac, G. Namkoong, Applied Sciences 2019, 9, 188.

[44] J. Idígoras, F. J. Aparicio, L. Contreras-Bernal, S. Ramos-Terrón, M. Alcaire, J. R. Sánchez-Valencia, A. Borras, Á. Barranco, J. A. Anta, ACS Appl. Mater. Interfaces 2018, 10, 11587.

[45] R. Wang, M. Mujahid, Y. Duan, Z.-K. Wang, J. Xue, Y. Yang, Advanced Functional Materials 2019, 1808843. DOI: 10.1002/adfm.201808843

[46] J. Ávila, C. Momblona, P. P. Boix, M. Sessolo, H. J. Bolink, Joule 2017, 1, 431.

[47] C. Aranda, C. Cristobal, L. Shooshtari, C. Li, S. Huettner, A. Guerrero, Sustainable Energy Fuels 2017, 1, 540. 
[48] G. Benecke, W. Wagermaier, C. Li, M. Schwartzkopf, G. Flucke, R. Hoerth, I. Zizak, M. Burghammer, E. Metwalli, P. Müller-Buschbaum, M. Trebbin, S. Förster, O. Paris, S. V. Roth, P. Fratzl, J Appl Cryst 2014, 47, 1797.

[49] J. Walton, P. Wincott, N. Fairley, A. Carrick, Peak Fitting with CasaXPS: A Casa Pocket Book, Accolyte Science, 2010.

[50] M. Mayer, SIMNRA User's Guide, Report IPP 9/113, Garching, 1997. 


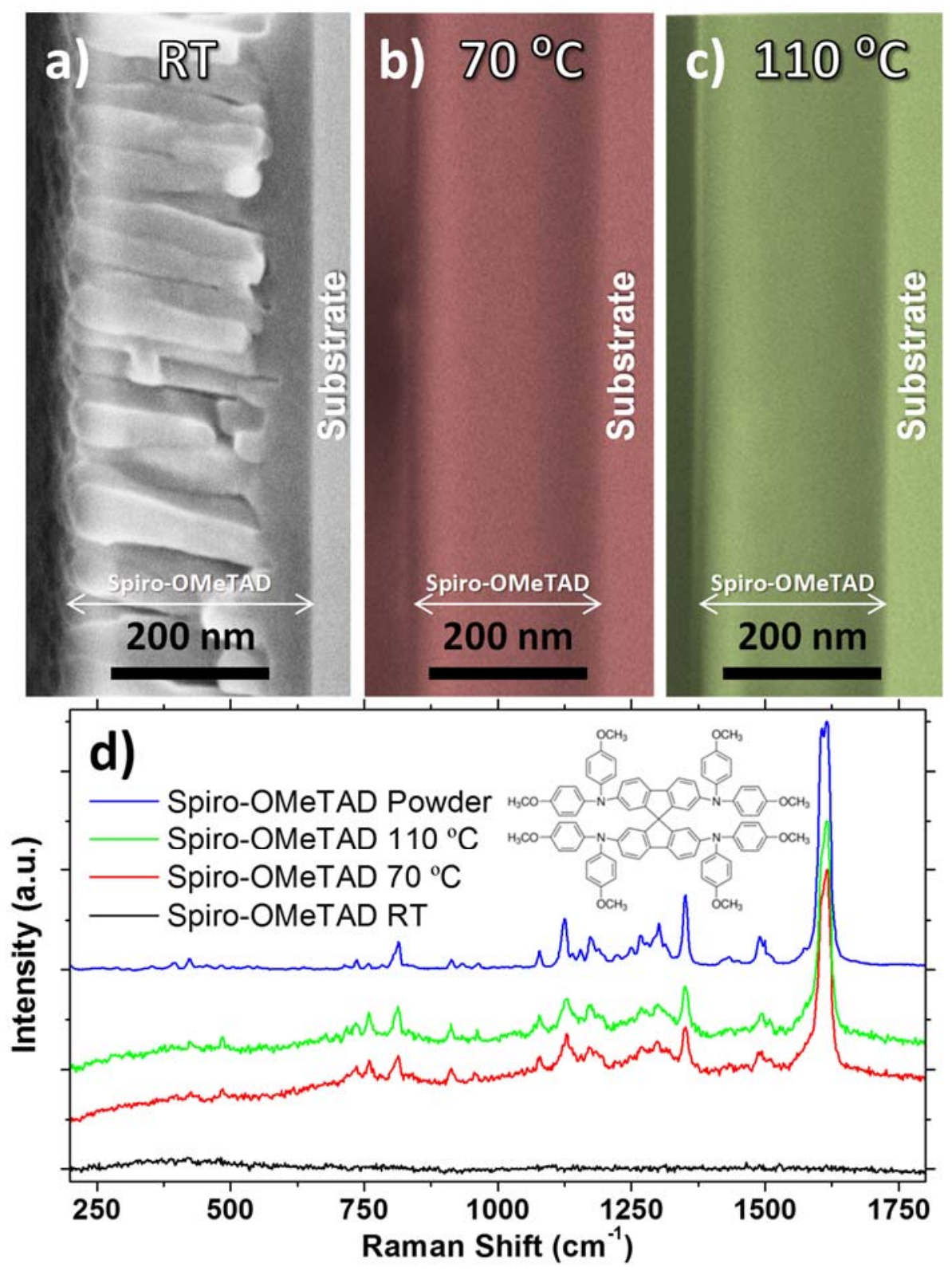

Figure 1. Microstructural and crystalline comparison. a-c) Cross-section SEM views of Spiro-OMeTAD molecules sublimated in vacuum on a silicon wafer at room temperature (a), 70 (b) and $110^{\circ} \mathrm{C}$ (c). d) Raman spectra of the Spiro-OMeTAD thin films showed in a-c) with the same colour code (black, red and green correspond to RT, 70 and $110^{\circ} \mathrm{C}$, respectively). The Raman spectrum of the Spiro-OMeTAD molecules in powder form has been added for comparison (blue line). 

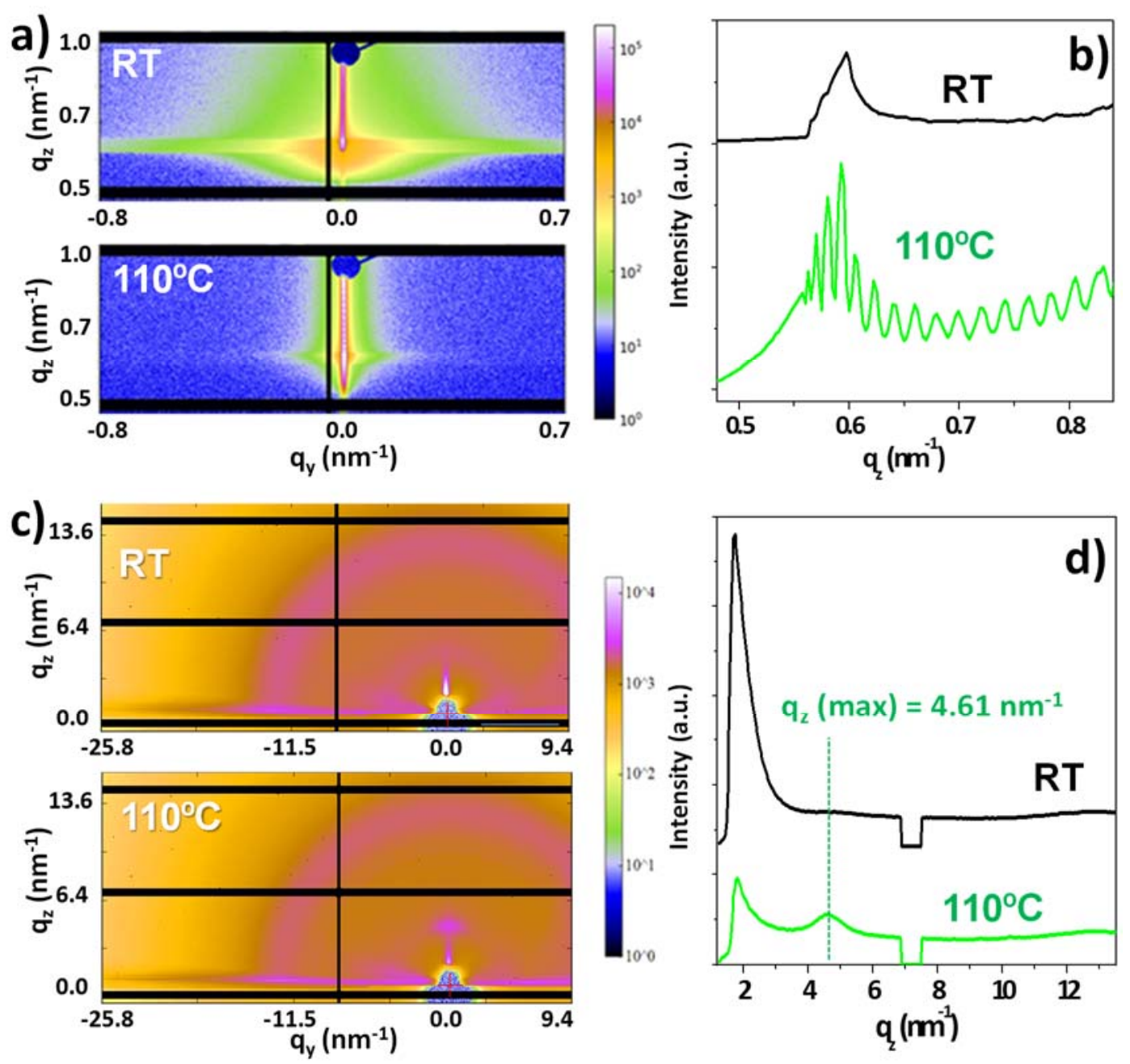

Figure 2. X-Ray Scattering Characterization. 2D GISAXS (a) and 2D GIWAXS (c) patterns for the vacuum sublimated Spiro-OMeTAD layers deposited on Si wafers at different temperatures. Out-of-plane 1D GISAXS (b) and Out-of-plane 1D GIWAXS (d) intensity profiles as a function of $\mathrm{q}_{\mathrm{z}}$. Guiding lines are added to identify the appearance of a new peak for the case of high deposition temperature in $\mathrm{d}$ ). 

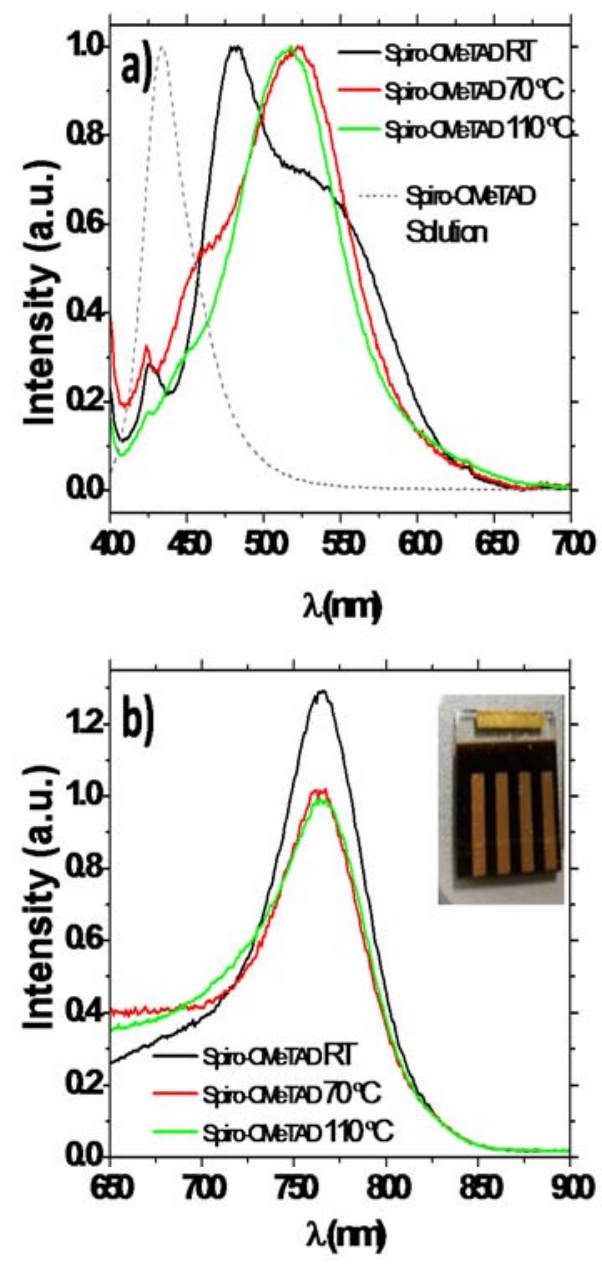

Figure 3. Photoluminescence study. a) Normalized steady-state photoluminescence spectra corresponding to the vacuum sublimated Spiro-OMeTAD layers (Excitation at $385 \mathrm{~nm}$ ) deposited at different temperatures. The spectrum of Spiro-OMeTAD molecules in chlorobenzene solution is also added for comparison. b) Photoluminescence spectra corresponding to the perovskite layers obtained by exciting with $490 \mathrm{~nm}$ light the complete solar cell through the glass. The spectra have been obtained by averaging the luminescence in different areas of the samples. The inset shows a photograph of a complete solar cell fabricated. 


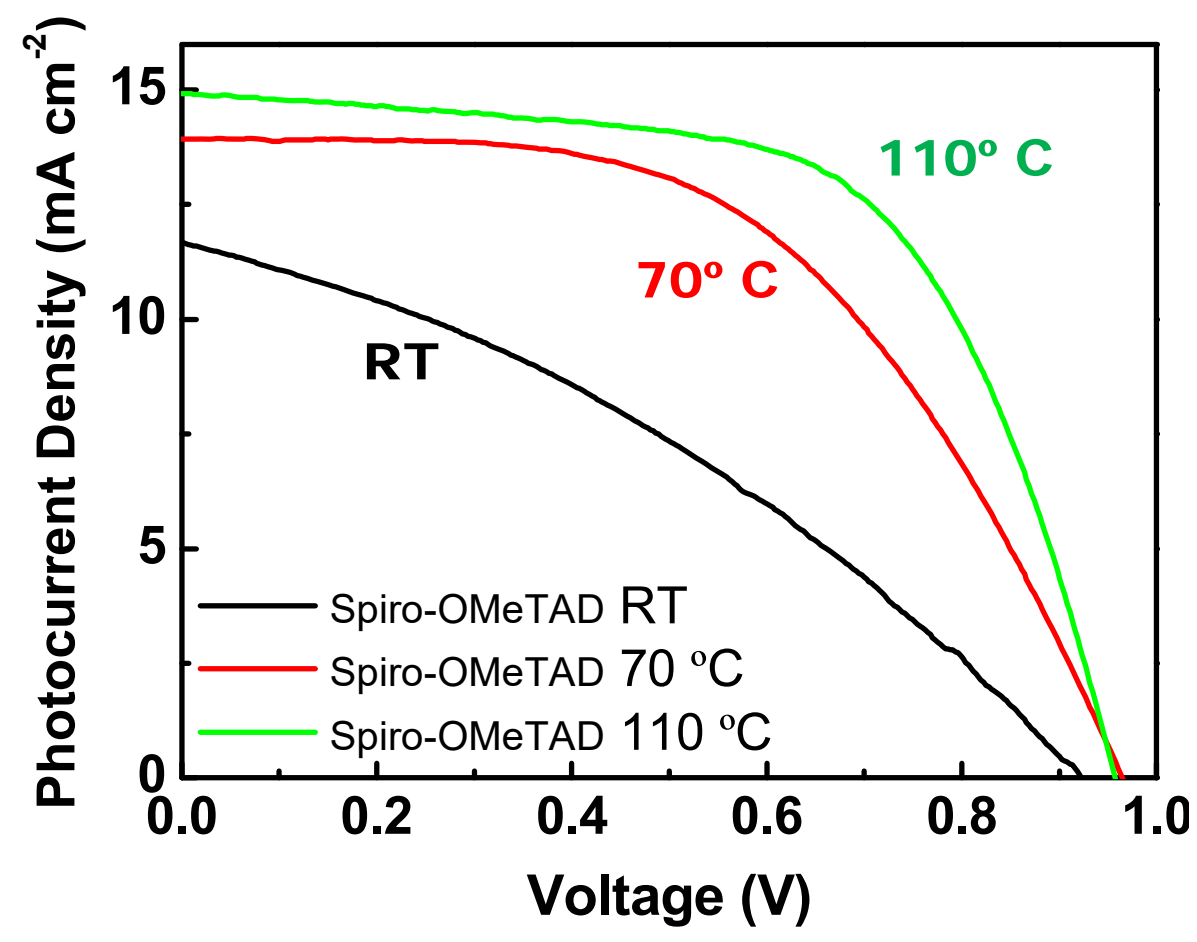

Figure 4. Photovoltaic properties. Photocurrent Density - Voltage characteristics of the champion solar cells containing vacuum sublimated Spiro-OMeTAD layers at RT (black), $70^{\circ}$ (red) and $110^{\circ} \mathrm{C}$ (green). 

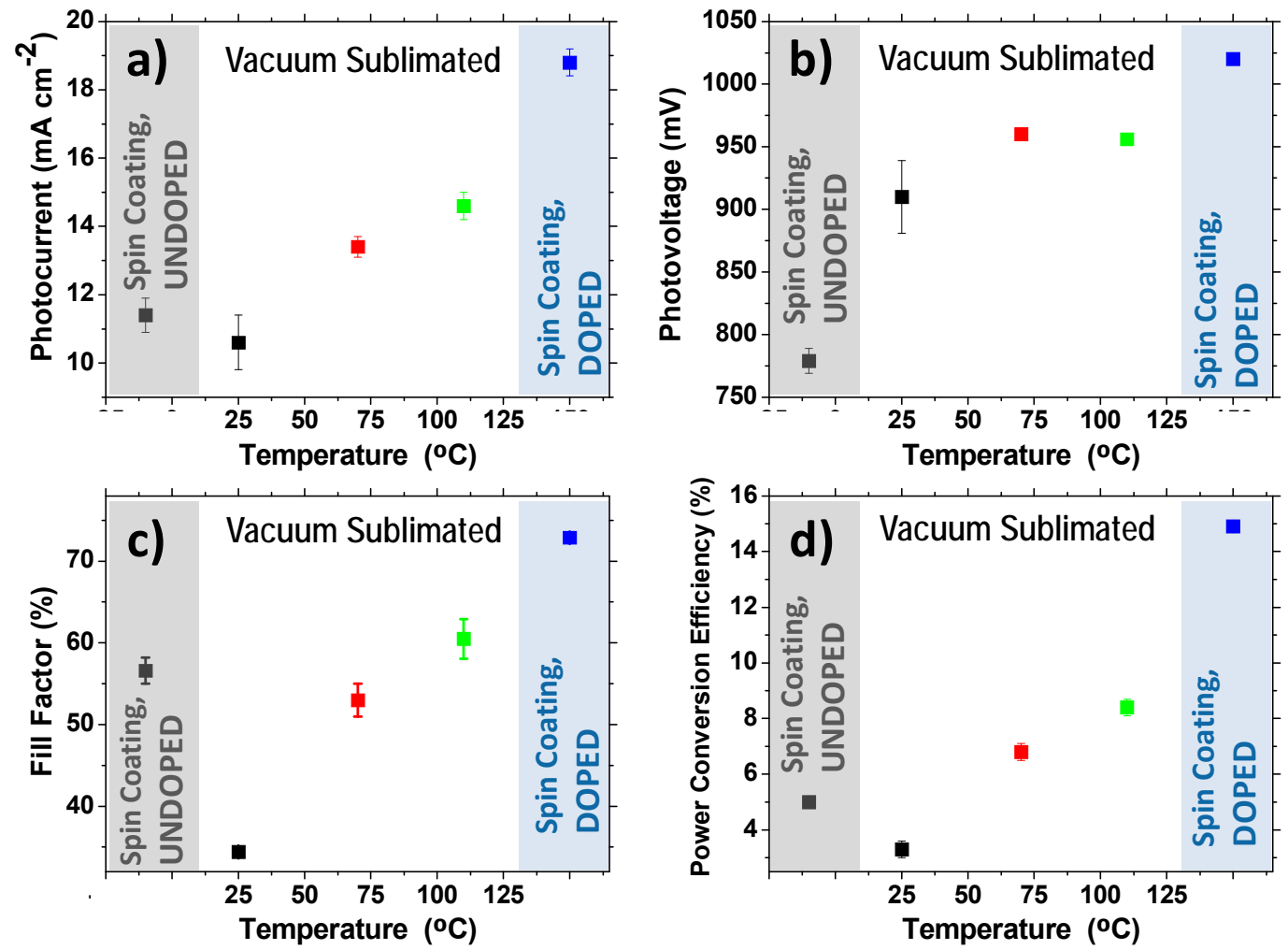

Figure 5. Photovoltaic parameters. a) Photocurrent, b) photovoltage, c) fill factor and d) power conversion efficiency of the solar cells incorporating vacuum sublimated SpiroOMeTAD layers at RT (black), $70^{\circ}$ (red) and $110^{\circ} \mathrm{C}$ (green). The graphs also show full solutionprocessed solar cells doped with LiTFSI and TBP (right) and dopant-free (left) Spiro-OMeTAD layers. 

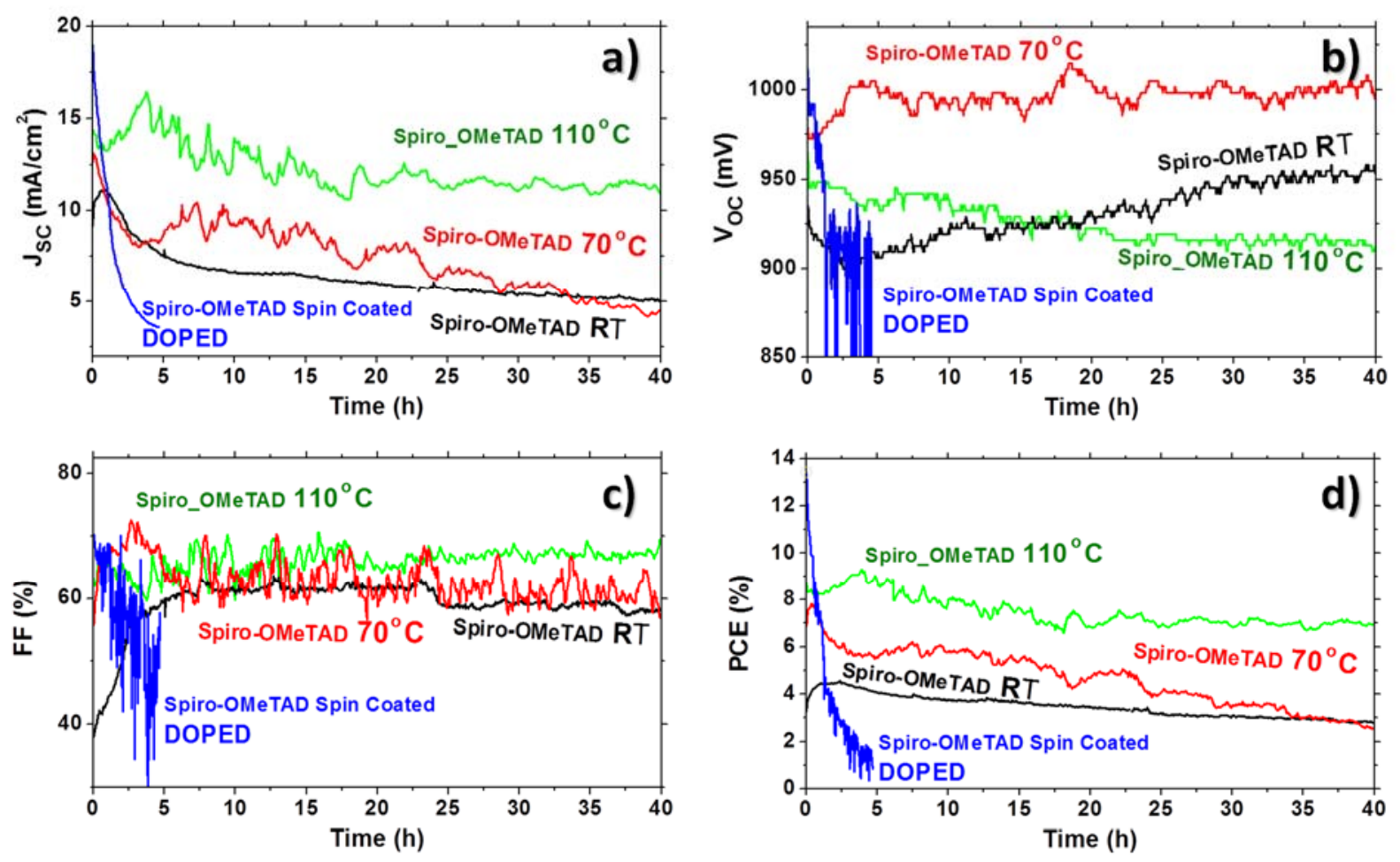

Figure 6. Stability study. a) Photocurrent, b) photovoltage, c) fill factor and d) power conversion efficiency evolution during 40 hours under continuous illumination of the solar cells incorporating vacuum sublimated Spiro-OMeTAD layers at RT (black), $70^{\circ}$ (red) and $110^{\circ} \mathrm{C}$ (green). The graphs also show full solution-processed solar cells incorporating spin-coated Spiro-OMeTAD layers doped with LiTFSI and TBP. 
Table 1. Elemental composition. extracted from the detailed XPS and RBS spectra shown in Figure S2 and S3 (See Experimental details)

\begin{tabular}{lllll}
\hline SAMPLE & $\mathbf{O} \%$ (XPS / RBS) & $\mathbf{C} \%$ (XPS / RBS) & N\% (XPS / RBS) & I\% (XPS / RBS) \\
\hline RT & $10.9 / 8.6$ & $84.6 / 85.7$ & $4.4 / 5.7$ & $<0.1 / 0$ \\
$\mathbf{1 1 0}{ }^{\circ} \mathbf{C}$ & $10.8 / 8.6$ & $84.9 / 85.7$ & $4.3 / 5.7$ & $0 / 0$ \\
Theoret. & 8.7 & 87.0 & 4.3 & 0 \\
\hline
\end{tabular}

Table 2. Main photovoltaic parameters. Short-circuit photocurrent $\left(\mathrm{J}_{\mathrm{SC}}\right)$, open circuit photovoltage (VOC), Fill Factor (FF) and Power Conversion Efficiency (PCE) of the solar cells containing the vacuum sublimated Spiro-OMeTAD layer at different temperatures (top rows). The photovoltaic parameters of a cell containing dopant-free and doped Spiro-OMeTAD layer prepared by spin coating have been added for comparison (bottom rows)

\begin{tabular}{rcccc}
\hline SAMPLE & $\mathbf{J}_{\mathrm{SC}}\left(\mathbf{m A} / \mathbf{c m}^{2}\right)$ & $\mathbf{V}_{\mathrm{OC}}(\mathbf{m V})$ & Fill Factor (\%) & PCE (\%) \\
\hline $\mathbf{R T}$ & $10.6 \pm 0.8$ & $910 \pm 29$ & $34.4 \pm 0.3$ & $3.3 \pm 0.3$ \\
$\mathbf{7 0}{ }^{\circ} \mathbf{C}$ & $13.4 \pm 0.3$ & $960 \pm 4$ & $53.0 \pm 2.0$ & $6.8 \pm 0.3$ \\
$\mathbf{1 1 0}{ }^{\circ} \mathbf{C}$ & $14.6 \pm 0.4$ & $956 \pm 2$ & $60.5 \pm 2.4$ & $8.4 \pm 0.3$ \\
\hline Dopant-free Spiro-OMeTAD & $11.4 \pm 0.5$ & $779 \pm 10$ & $56.6 \pm 1.6$ & $5.0 \pm 0.1$ \\
spin-coating & & & $72.9 \pm 0.4$ & $14.9 \pm 0.2$ \\
\hline Doped (LiTFSI, TBP)Spiro-OMeTAD & $18.8 \pm 0.4$ & $1020 \pm 4$ & \\
spin-coating & & &
\end{tabular}


Spiro-OMeTAD, the most used solid-state hole conductor in perovskite solar cells, is usually processed by solution but needs the presence of dopants for efficient charge transport.

Here, dopant-free Spiro-OMeTAD layers prepared vacuum sublimation are reported. Temperature control of the samples during evaporation induces microstructural changes and crystalline growth. The implementation in perovskite solar cells demonstrates unprecedented superior stability with respect to the solution-processed, by using the most widely used SSHC in the literature. 


\section{Supporting Information}

Enhanced stability of perovskite solar cells incorporating dopant-free crystalline SpiroOMeTAD layers by vacuum sublimation

Angel Barranco ${ }^{*}$ Maria C. Lopez-Santos, Jesus Idigoras, Francisco J. Aparicio, José Obrero-Pérez, Victor Lopez-Flores, Lidia Contreras-Bernal, Victor Rico, Javier Ferrer, Juan P. Espinos, Ana Borras, Juan A. Anta, Juan R. Sanchez-Valencia*.

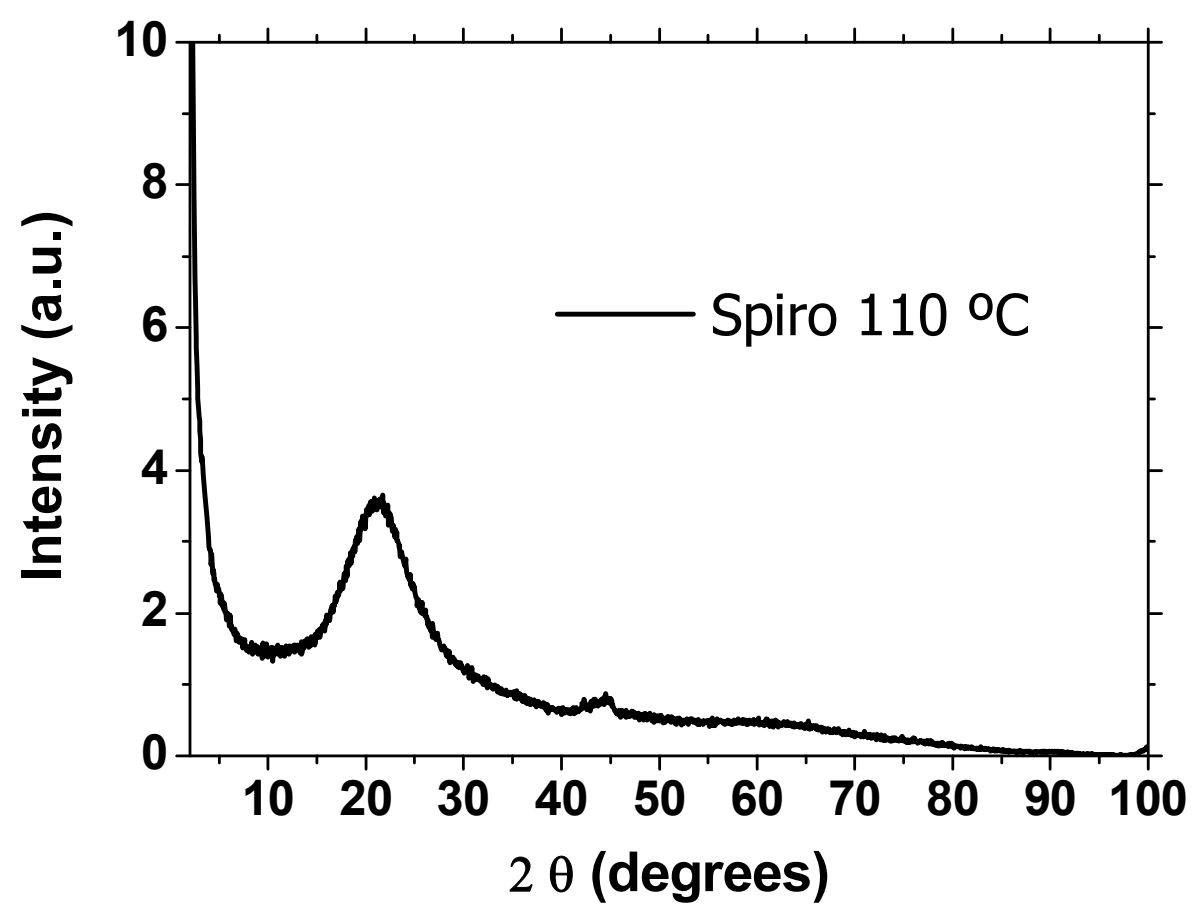

Figure S1. Grazing Incidence X-Ray Diffraction. GI-XRD of the Spiro-OMeTAD layer deposited at $110^{\circ} \mathrm{C}$. 

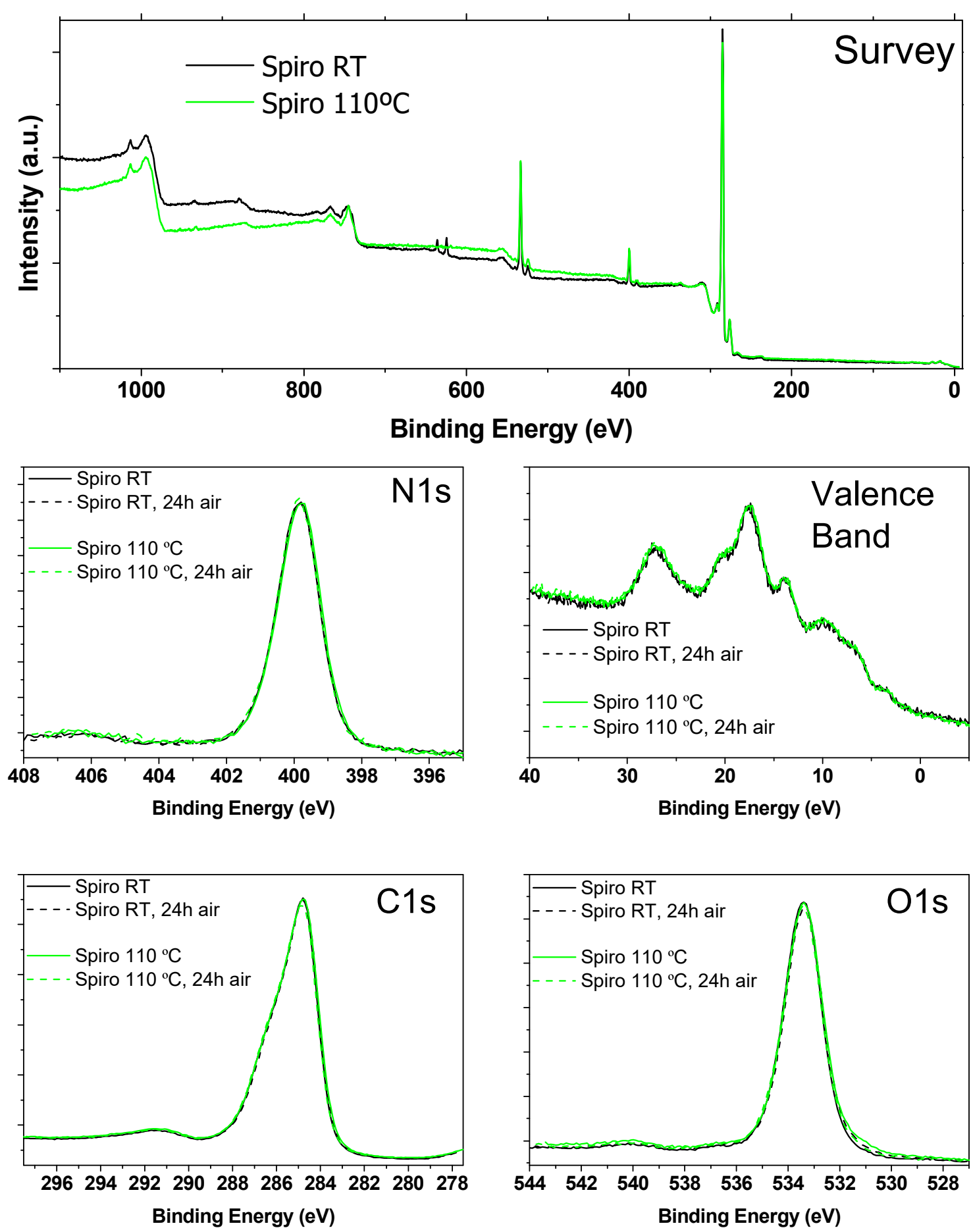

Figure S2. X-Ray Photoelectron Spectroscopy. a) Survey spectrum. Core level detailed spectrum of C1s (b), O1s (c) and N1s (d), and valence band (e) for Spiro-OMeTAD samples deposited at $\mathrm{RT}$ and $110^{\circ} \mathrm{C}$ before and after exposing the samples to the atmosphere during $24 \mathrm{~h}$. It is worth to remark that the spectra are identical in the four cases. 

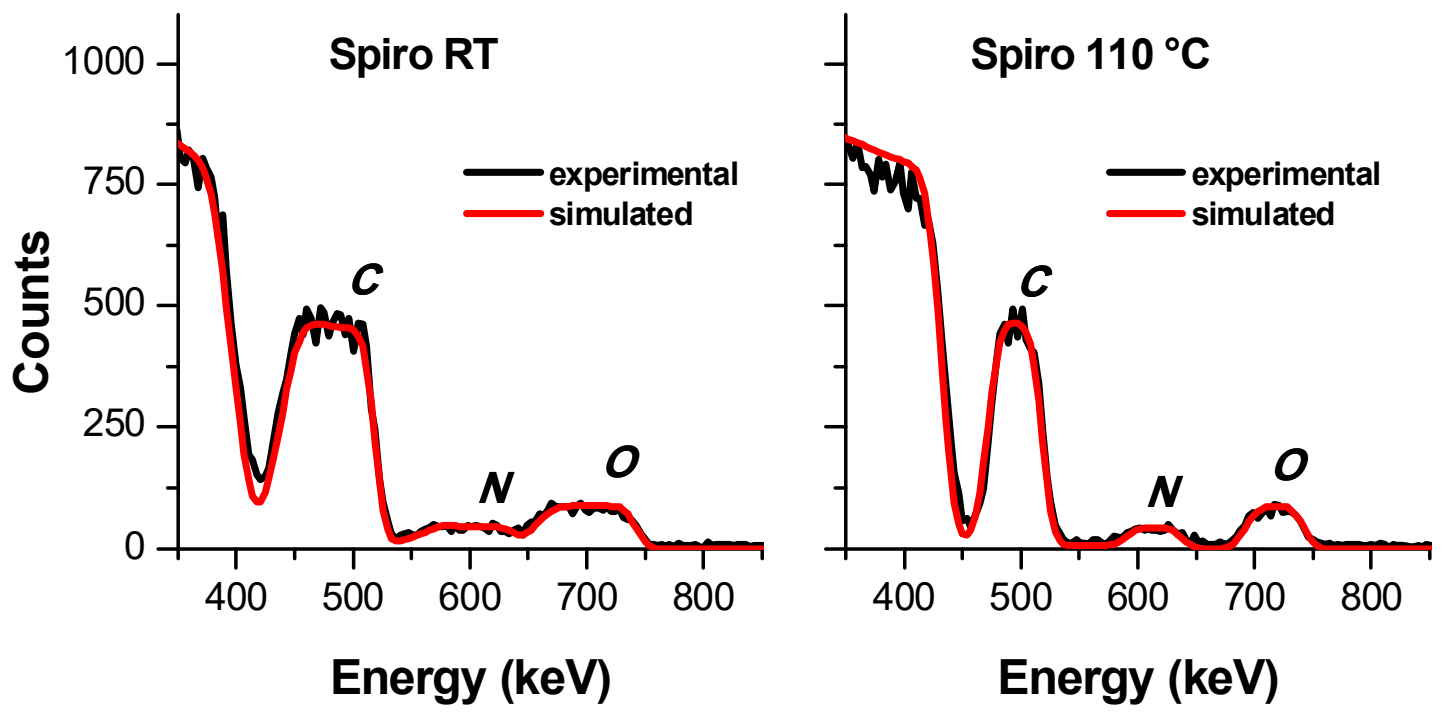

Figure S3. Rutherford Backscattering Spectra. Experimental and simulated spectra RBS with 2.0 alpha particles and PIPS detector set at $165^{\circ}$ of Spiro-OMeTAD deposited at RT and $110^{\circ} \mathrm{C}$.

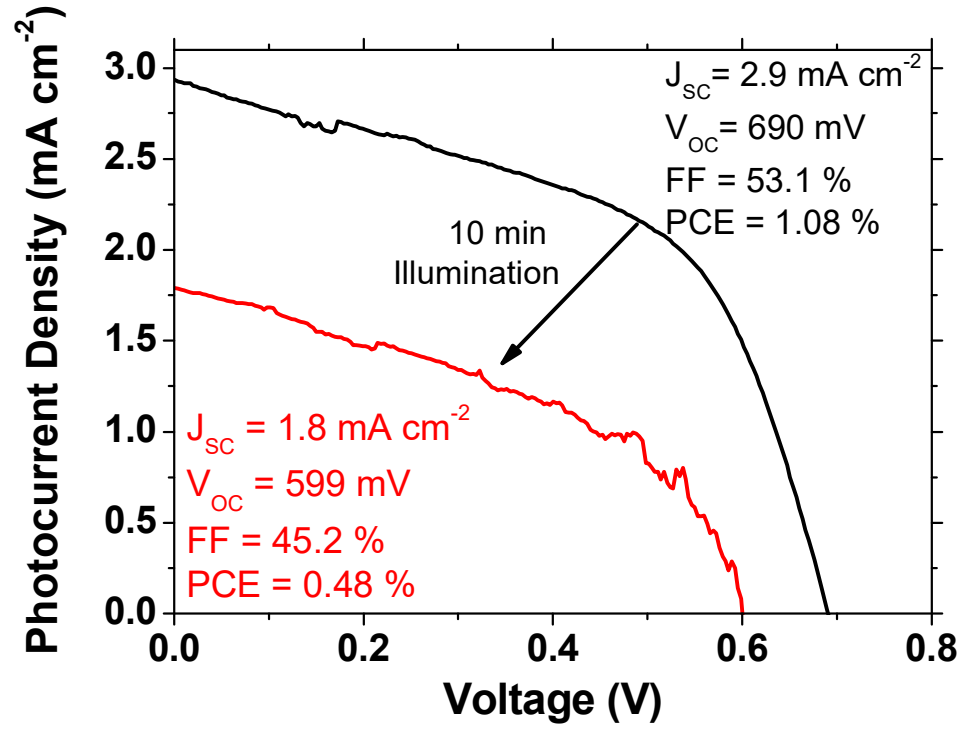

Figure S4. Photocurrent Density-Voltage curves for solar cells without the Hole Transporting Layer (Gold layer deposited on top of the perovskite). The curve after 10 minutes of continuous illumination (red curve) shows very poor stability. 

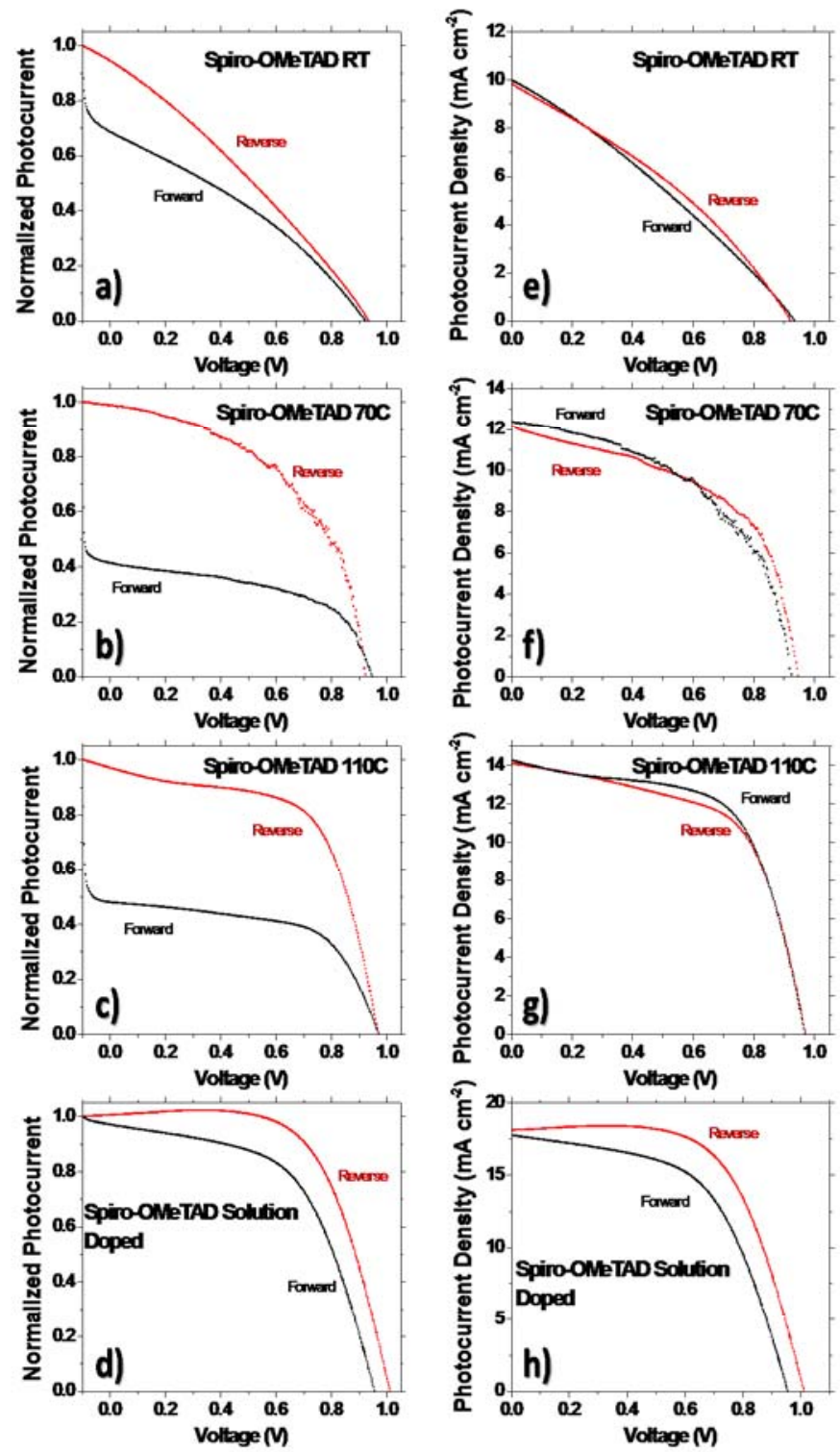

Figure S5. Photocurrent Density-Voltage in forward (black dots) and reverse (red dots) scans. The curves are presented normalized to the maximum photocurrent at $-0.1 \mathrm{~V}$ (a-d) and without normalization $(\mathrm{e}-\mathrm{h})$ for solar cells incorporating vacuum sublimated Spiro-OMeTAD layers at RT $(\mathrm{a}, \mathrm{e}), 70^{\circ} \mathrm{C}(\mathrm{b}, \mathrm{f})$ and $110^{\circ} \mathrm{C}(\mathrm{c}, \mathrm{g})$ and spin-coated Spiro-OMeTAD (doped) $(\mathrm{d}, \mathrm{h})$. 


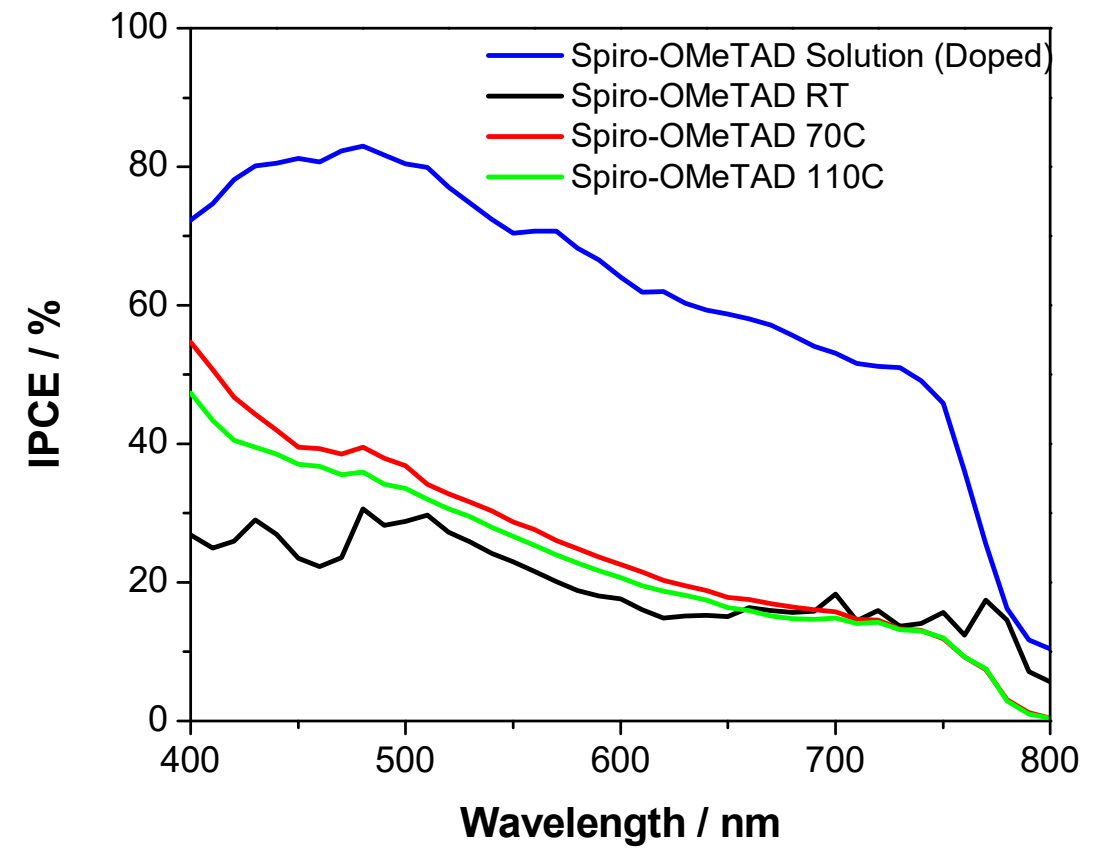

Figure S6. IPCE curves for solar cells incorporating vacuum sublimated Spiro-OMeTAD layers at RT (black), $70^{\circ} \mathrm{C}$ (red) and $110^{\circ} \mathrm{C}$ (gree) and spin-coated Spiro-OMeTAD (doped) (blue).
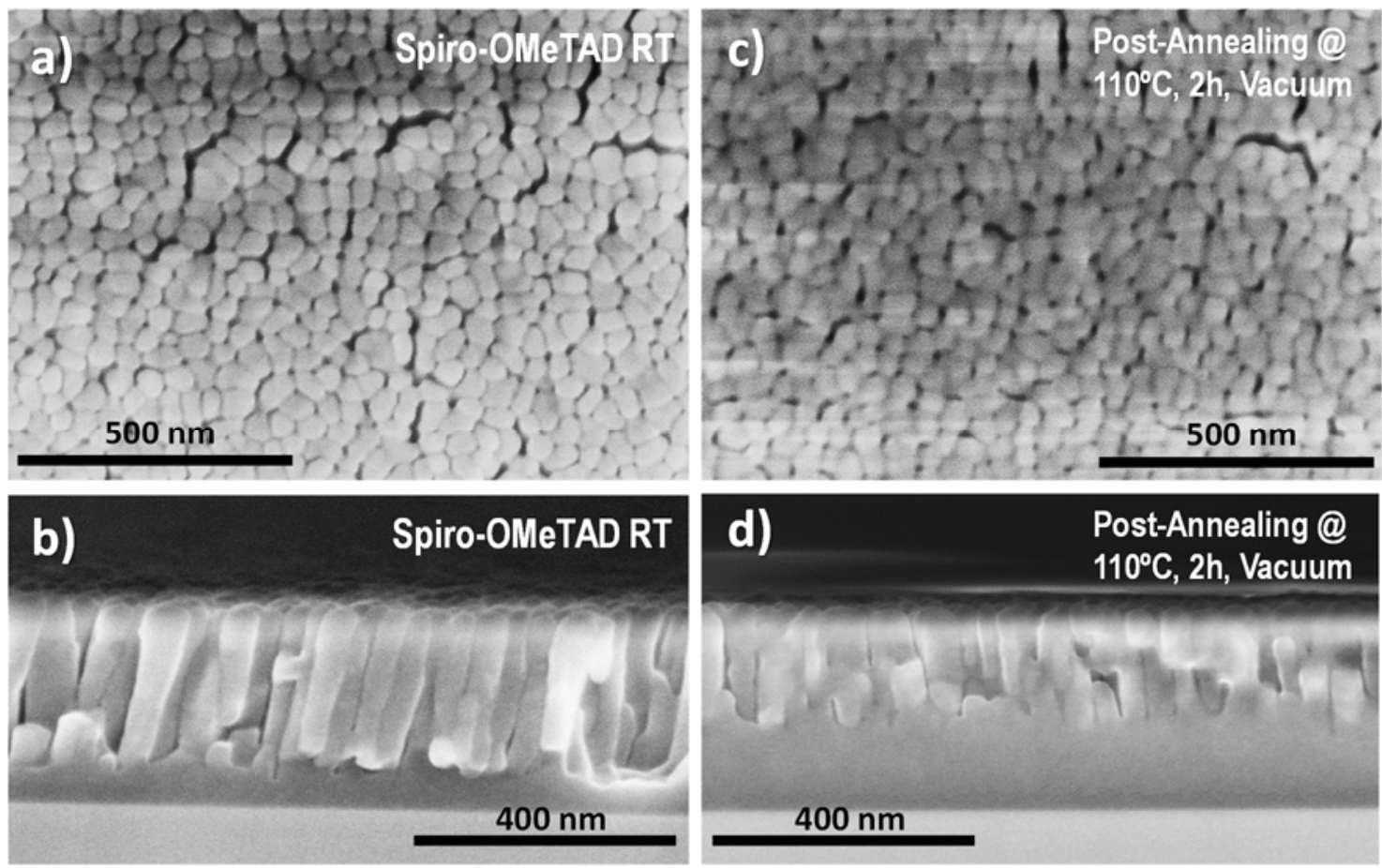

Figure S7. Top (a, c) and cross-section (b,d) SEM views of the Spiro-OMeTAD layers sublimated onto a silicon wafer at room temperature $(a, b)$ and post-annealed at $110^{\circ} \mathrm{C}$ during 2 hours in vacuum $(\mathrm{c}, \mathrm{d})$. 


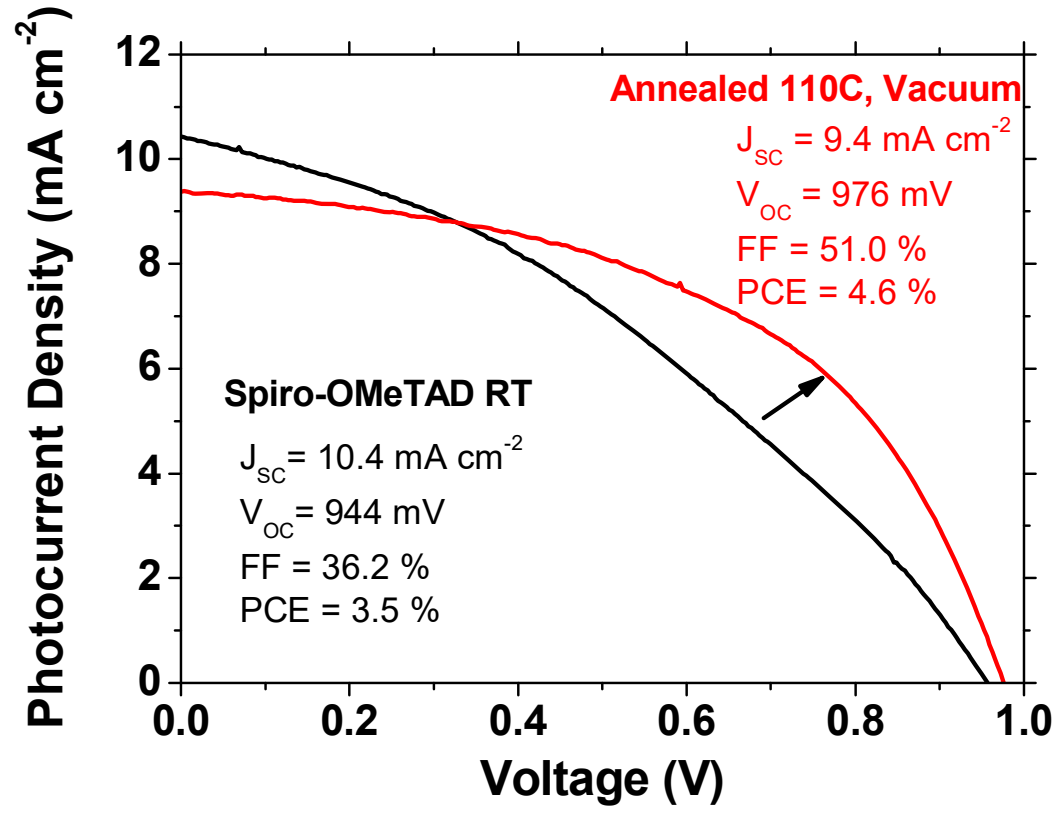

Figure S8. Photocurrent Density-Voltage curves for solar cells incorporating vacuum sublimated Spiro-OMeTAD layers at RT (black curve) and post-annealed at $110^{\circ} \mathrm{C}$ during 2 hours in vacuum (red curve). 

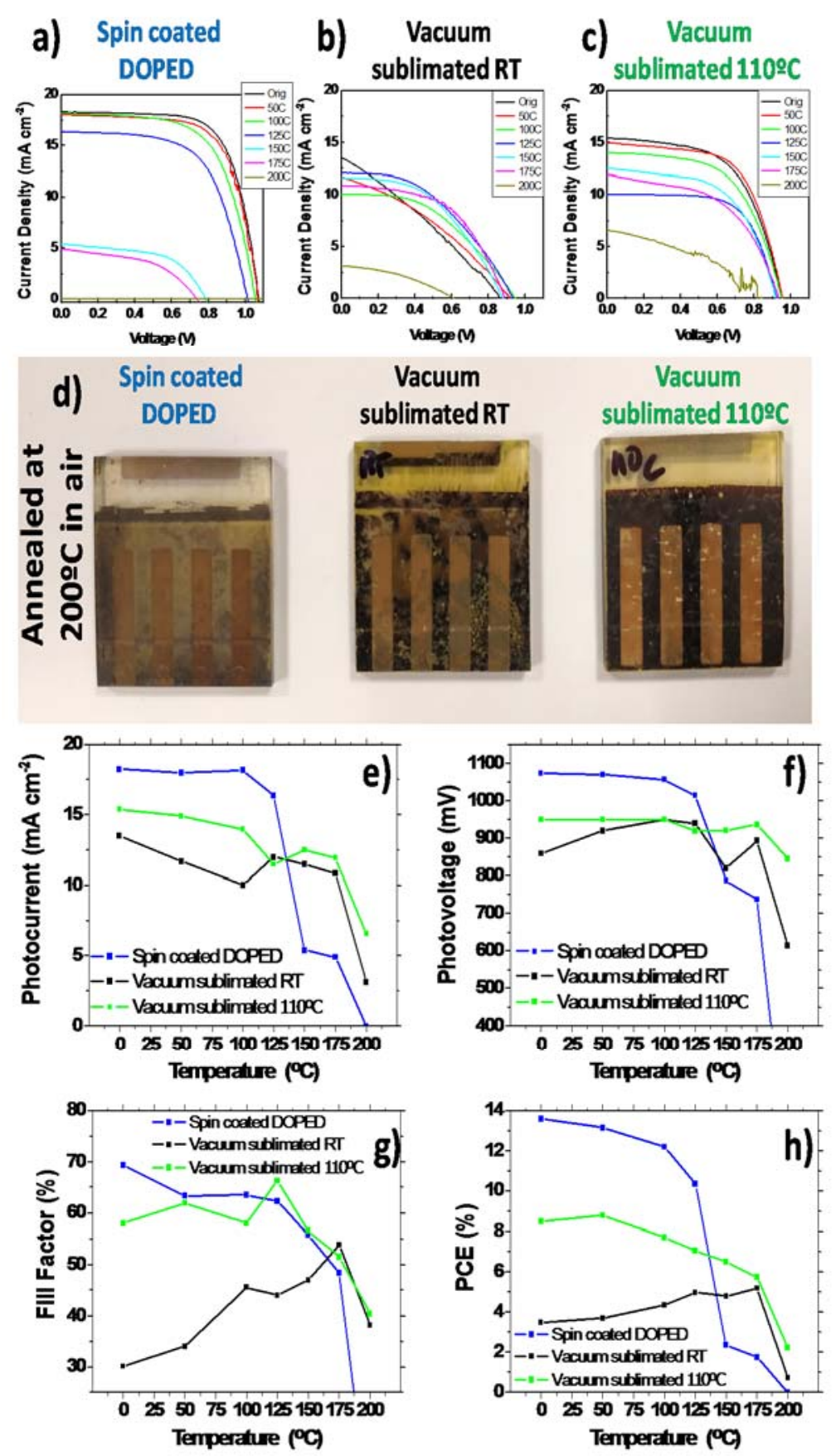

Figure S9. a-c) Photocurrent Density - Voltage characteristics of the champion solar cells containing solution-processed (a) and vacuum sublimated Spiro-OMeTAD layers at RT (b), and $110^{\circ} \mathrm{C}(\mathrm{c})$, after annealing treatments in air during 30 minutes at the indicated temperatures. d) Photograph of the samples studied after annealing at $200^{\circ} \mathrm{C}$ in air. e-h) Photovoltaic parameters, photocurrent (e), photovoltage (f), fill factor (g) and PCE (h), corresponding to the $\mathrm{J}-\mathrm{V}$ curves shown in $\mathrm{a}-\mathrm{c})$. 


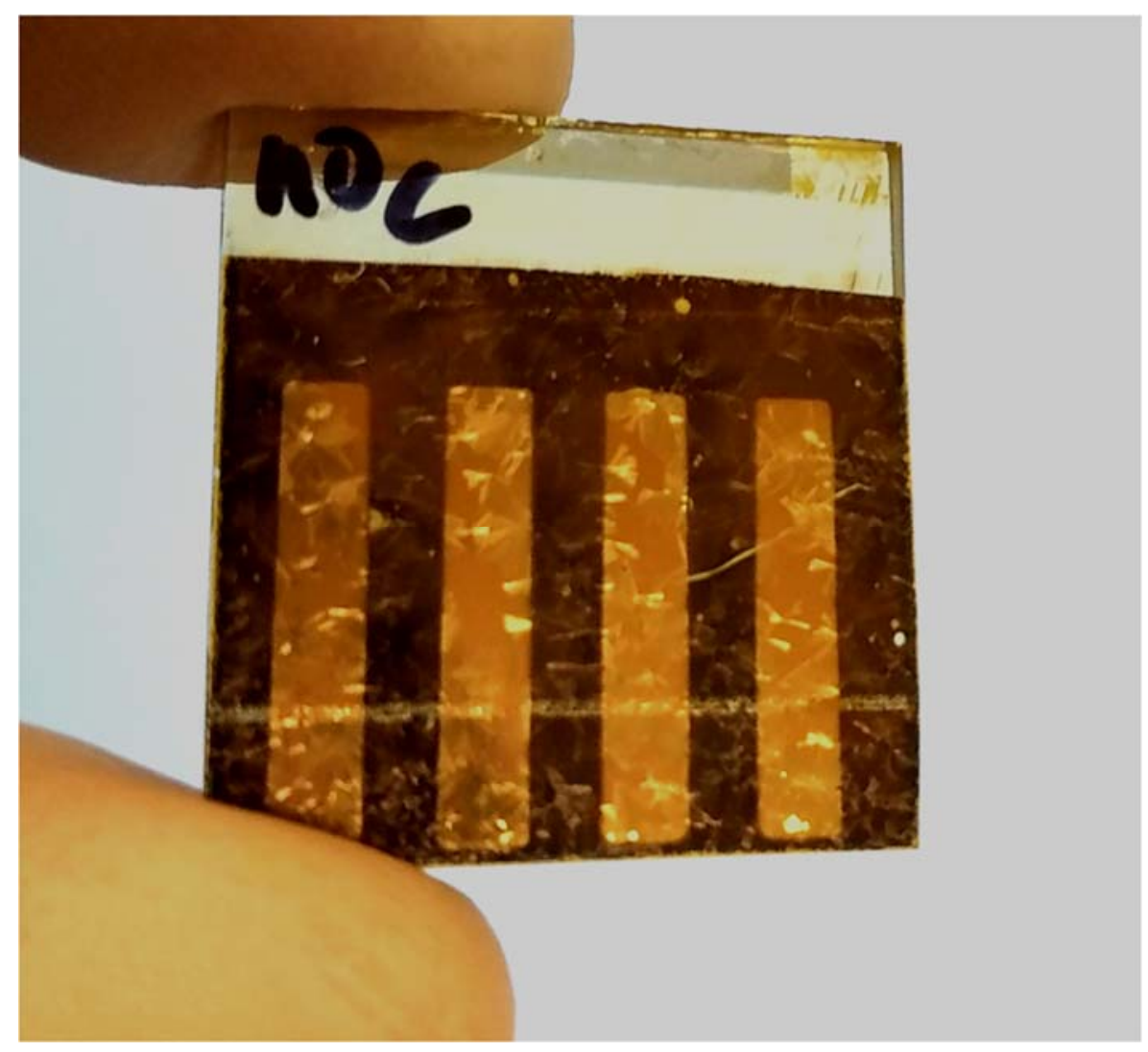

Figure S10. Close-up photograph of the solar cell implementing vacuum sublimated SpiroOMeTAD layer after annealing at $200^{\circ} \mathrm{C}$ in air, where it can be seen a macroscopic crystalline growth. 